\title{
Facteurs environnementaux associés au trouble du spectre de l'autisme : étude de délimitation portant sur les années 2003 à 2013
}

Michelle Ng, M.P.H. (1,2); Joanne G. de Montigny, M.H.A. (3); Marianna Ofner, Ph. D. (1,2); Minh T. Do, Ph. D. (1,2)

Cet article a fait l'objet d'une évaluation par les pairs.

\section{Résumé}

Introduction : Le nombre d'enfants chez lesquels on diagnostique un trouble du spectre de l'autisme (TSA) grimpe rapidement depuis une décennie. L'étiologie de ce trouble est toutefois en grande partie inconnue, même si la contribution de l'environnement est importante par rapport à celle de la génétique. Nous avons procédé à une étude de délimitation pour évaluer en détail l'état actuel des connaissances sur les facteurs environnementaux présents depuis le stade de la préconception jusqu'au début de la vie que l'on associe au TSA et pour dégager les lacunes de la recherche.

Méthodologie : Nous avons cherché dans les bases de données électroniques MEDLINE, PsycINFO et ERIC des articles portant sur des facteurs de risque potentiels ou des facteurs de protection des environnements physiques et sociaux associés au TSA et à ses sous-catégories entre le $1^{\text {er }}$ janvier 2003 et le 12 juillet 2013. Nous avons regroupé les articles en thèmes généraux en fonction de l'exposition environnementale : facteurs chimiques, physiologiques, nutritionnels et sociaux.

Résultats : Nous avons trouvé plus de 50000 publications, mais après élimination des études inadmissibles il est resté 315 articles. La plupart de ces études portaient sur les facteurs psychologiques, suivis de près par les facteurs chimiques et, à un degré beaucoup moindre, les facteurs nutritionnels et sociaux associés au TSA. En dépit d'une masse importante de publications et de nombreuses études hétérogènes, quelques facteurs de risque se sont démarqués régulièrement : facteurs chimiques comme les polluants atmosphériques causés par la circulation; facteurs physiologiques, dont l'âge avancé des parents, les naissances prématurées, l'insuffisance de poids à la naissance, l'hyperbilirubinémie et les grappes de complications de la grossesse et enfin le statut de la mère vis-à-vis de l'immigration. En dépit de recherches poussées sur les vaccins, les faits révèlent de façon écrasante que rien n'appuie l'existence d'un lien avec le TSA.

Conclusion : Le manque d'uniformité, de temporalité et de spécificité des liens entre les facteurs environnementaux et le TSA demeure l'obstacle le plus important dans l'établissement de liens de cause à effet. Une recherche plus robuste s'impose pour supprimer le manque d'uniformité dans les publications. Les recherches futures devraient porter sur des mécanismes sous-jacents des liens entre facteurs de risque que nous avons identifiés et le TSA.

Mots-clés : TSA, trouble du spectre de l'autisme, autisme, exposition environnementale, étiologie

\section{Introduction}

Le trouble du spectre de l'autisme (TSA) s'entend d'un groupe de troubles du développement neurologique dont la gravité varie sur les plans du déficit de la communication et de l'interaction sociale et avec des intérêts, des activités et des
Diffuser cet article sur Twitter

Points saillants

- Les facteurs physiologiques et chimiques sont les facteurs environnementaux les plus souvent étudiés à l'égard du TSA. La recherche sur les facteurs nutritionnels et sociaux est limitée.

- Des parents plus âgés, une insuffisance de poids à la naissance, la prématurité, la jaunisse du nouveau-né et les grappes de complications de la grossesse ont été régulièrement associés au TSA.

- On commence à dégager des éléments de preuve sur le lien entre les polluants atmosphériques causés par la circulation et le TSA.

- Un lien constant a été établi entre le statut vis-à-vis de l'immigration de la mère et le TSA.

- Le manque d'uniformité, de temporalité et de spécificité des liens entre les facteurs environnementaux et le TSA demeure l'obstacle le plus important à l'établissement de liens de cause à effet.

comportements répétitifs limités ${ }^{1}$. Le nombre d'enfants avec un TSA augmente rapidement depuis une décennie ${ }^{2}$. Aux États-Unis, le nombre de cas a augmenté de $123 \%$ entre 2002 et 2010 et l'on en estime la prévalence à 1 enfant de huit ans sur $68^{3}$. Au Canada, entre 2003 et 2010, on a signalé des augmentations de $70 \%$ à l'Île-duPrince-Édouard et de $95 \%$ dans le sud-est de l'Ontario et une prévalence correspondante en 2010 d'un enfant de 6 à 9 ans sur 106 et d' 1 sur $63^{4}$. Une plus grande sensibilisation au TSA et des modifications des 
critères de diagnostic peuvent avoir contribué à cette augmentation ${ }^{5}$, mais ces facteurs expliquent une partie seulement de la montée de la prévalence ${ }^{6,7}$. Même si la contribution de la génétique à l'étiologie du TSA est connue ${ }^{8}$, la montée rapide de la prévalence du TSA ne peut être attribuée complètement à la génétique seule.

Une étude de concordance jumelée a montré que les facteurs environnementaux communs causent $58 \%$ de la variation de la sensibilité au TSA ${ }^{8}$. De plus, on sait que la période prénatale et celle de la petite enfance constituent des périodes cruciales de la croissance au cours desquelles les enfants sont particulièrement vulnérables aux effets nocifs des dangers environnementaux qui peuvent causer des maladies infantiles ${ }^{9}$. Le rôle des facteurs environnementaux dans l'apparition du TSA demeure toutefois en grande partie inconnu. Aucune des études portant sur des facteurs de risque possibles de TSA réalisées jusqu'à maintenant ${ }^{10-16}$ n'a analysé en détail la portée complète de la contribution de l'environnement au TSA.

La présente étude de délimitation vise à évaluer en détail l'état actuel des connaissances sur les facteurs environnementaux associés à l'incidence du TSA et à dégager les lacunes de la recherche. Nous visons plus précisément à déterminer toute exposition environnementale en lien avec le TSA, que ce soient les facteurs chimiques, physiologiques, nutritionnels ou sociaux, et ce, depuis la période qui précède la conception jusqu'à celle du début de la vie.

\section{Méthodologie}

Cet examen a suivi le cadre de délimitation d'Arksey et O'Malley ${ }^{17}$. Nous avons cherché dans les bases de données électroniques MEDLINE, PsycINFO et ERIC des études principales ou des revues de la littérature portant sur les facteurs de risque modifiables potentiels ou sur des facteurs de protection provenant de l'environnement physique ou social associés au TSA. Nous avons dressé une liste détaillée de rubriques et de mots clés médicaux avec l'aide d'un bibliothécaire de l'Université d'Ottawa. Les termes de recherche visaient deux concepts principaux : (1) le TSA et ses sous-catégories, dont le trouble envahissant du développement non spécifié (TED-NS), l'autisme et le syndrome d'Asperger et (2) les expositions ou facteurs de risque environnementaux, l'état de la mère avant et pendant la grossesse et l'état du père avant la conception. Nous avons adapté la stratégie de recherche à d'autres bases de données, soit PsycINFO et ERIC, en utilisant leurs rubriques correspondantes. Les recherches ont été limitées aux articles comportant un résumé publié en anglais entre le $1^{\text {er }}$ janvier 2003 et le 12 juillet 2013, afin de concentrer la recherche sur les publications actuelles. Nous avons exclu les articles si les liens épidémiologiques qu'ils décrivaient comportaient des comorbidités, les conséquences d'une vie avec le TSA ou des voies biochimiques non liées directement à l'étiologie. Nous aussi exclu les études utilisant des modèles animaux, les études cellulaires et des études strictement génétiques. Nous avons aussi filtré les commentaires, les éditoriaux, les lettres, les articles d'actualité et ceux qui ne portaient pas principalement sur l'étiologie du TSA.

Nous avons classé les articles de revues en thèmes généraux : facteurs chimiques, physiologiques, nutritionnels, sociaux et autres, en fonction de la nature de l'exposition à l'environnement analysé. Dans chaque grand thème, nous avons dégagé des sous-thèmes. Nous avons fait un résumé incluant l'année de publication, la conception de l'étude, la population à l'étude, l'exposition, les facteurs confusionnels, la définition des cas et les principales constatations que nous avons tirées de la version intégrale de chaque article. Notre processus d'évaluation a permis de dégager 315 articles pour analyse finale (figure 1). Nous avons ciblé principalement les études originales et les examens systématiques.

\section{Résultats}

La littérature englobait un vaste éventail de recherches portant sur les facteurs de risque environnementaux potentiellement associés au TSA. Des recherches ont été conduites dans le monde entier, mais de nombreuses études ont été réalisées dans les pays nordiques, aux États-Unis, au Royaume-Uni, en Australie et au Japon. La plupart des études ont porté sur des facteurs physiologiques, suivis de près par des facteurs chimiques puis des facteurs nutritionnels et sociaux associés au TSA (tableau 1 et figure 2). Ces domaines se chevauchaient, car plusieurs études ont porté sur une combinaison de facteurs : environ $14 \%$ des articles (44/315) ont exploré plus d'un thème. Le pourcentage le plus important d'études a porté sur les facteurs chimiques et nutritionnels (exposition aux métaux lourds et carences en minéraux). La figure 3 présente la fréquence des articles publiés en fonction du domaine de recherche et de la période de publication. La plupart des articles sont parus entre 2009 et 2013, sauf ceux sur les vaccins, surtout publiés entre 2003 et 2008.

\section{Dimension chimique}

Les facteurs chimiques analysés en lien avec le TSA comprenaient des substances chimiques environnementales, des vaccins, des médicaments et l'abus de substances. Les articles sur l'exposition aux substances chimiques de l'environnement et aux vaccins (principalement sur l'exposition postnatale dans les deux cas) ont compté pour environ $40 \%$ de la dimension chimique (figure 2).

\section{Substances chimiques de l'environnement}

Les substances chimiques de l'environnement analysées dans le contexte du TSA étaient principalement les métaux lourds, qui ont été trouvés dans des échantillons biologiques prélevés chez les enfants, suivis des polluants atmosphériques. Dans les deux cas, l'analyse s'est déroulée dans le contexte d'études cas-témoins (tableau 2). Quelques études ou examens ont porté sur les liens entre l'exposition prénatale ou périnatale et des substances chimiques ${ }^{18,19}$ et antiparasitaires ${ }^{20,21}$ utilisées au travail.

\section{Métaux lourds}

Beaucoup d'études ont analysé les biomarqueurs de l'exposition postnatale aux métaux lourds, en particulier le mercure, chez les enfants vivant avec le TSA ou l'autisme comparativement aux enfants sans TSA. L'évaluation des dents du bébé, en particulier de l'émail dentaire, qui commence à prendre forme dans l'utérus et continue de se former jusqu'à un an après la naissance ${ }^{22}$, peut aussi aider à déterminer l'exposition prénatale. Les cinq métaux lourds les plus étudiés étaient le mercure, le plomb, le cadmium, l'aluminium et l'arsenic.

\section{Mercure : biomarqueurs et sources d'exposition}

Le mercure a beaucoup attiré l'attention à cause des similitudes entre les symptômes de l'hydrargisme et ceux de l'autisme ${ }^{23}$. On a analysé les biomarqueurs de l'exposition au mercure chez les enfants atteints d'autisme principalement en mesurant les concentrations dans les cheveux (14 études), 
FIGURE 1

Processus de recherche et de sélection d'études publiées entre 2003 et 2013 sur les troubles du spectre de l'autisme, dans trois bases de données électroniques

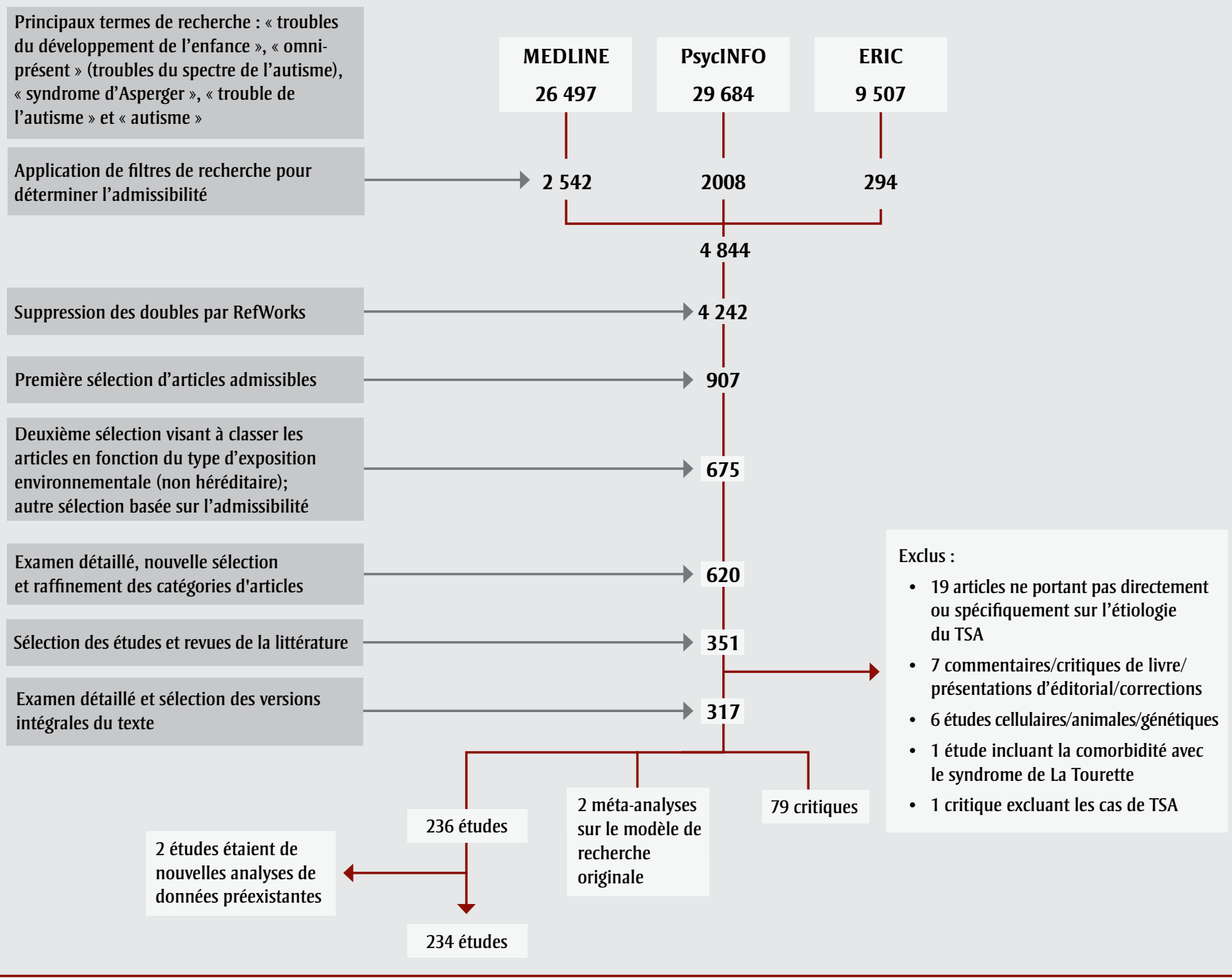

le sang (4), l'urine (4), les dents (2) et les ongles (1). Les résultats d'analyse manquaient toutefois en grande partie d'uniformité (tableau 3). On a aussi étudié les porphyrines urinaires, intermédiaires de la biosynthèse des thèmes, comme biomarqueurs possibles de l'exposition au mercure chez les personnes vivant avec l'autisme, car on a établi un lien entre l'élévation de la concentration de porphyrine et une exposition prolongée au mercure $^{24}$. On a découvert des concentrations élevées de porphyrines chez les enfants vivant avec l'autisme comparativement à ceux vivant sans autisme ${ }^{25-29}$, et les concentrations pourraient augmenter en fonction de la gravité des symptômes d'autisme ${ }^{30,31}$. Même si presque toutes les études ont attribué ces constatations à l'exposition au mercure, aucune n'a mesuré vraiment cette exposition sauf dans un cas, où l'on n'a constaté aucun lien avec le TSA, même si l'on a découvert un lien entre l'élévation des concentrations de porphyrines et le $\mathrm{TSA}^{27}$.

Quelques études portant sur l'exposition au mercure visées par notre examen s'intéressaient à d'autres sources que le thimérosal dans les vaccins et les immunoglobulines Rh. Des études écologiques ont démontré l'existence d'un lien entre des émissions de mercure dans l'environnement et des augmentations importantes des taux d'autisme ${ }^{32}$, qui peuvent être liées à la distance entre le domicile et les sources de pollution par le mercure comme des installations industrielles ou des centrales d'énergie $^{33}$. D'autres études ont porté sur des sources d'exposition prénatale et postnatale au mercure en lien avec le TSA, comme les amalgames dentaires de la mère ${ }^{27,34,35}$ et la consommation par la mère ou l'enfant de produits de la $\operatorname{mer}^{27,36,37}$, mais les résultats manquent d'uniformité.

Une revue systématique de la littérature réalisée en 2012 a conclu qu'à cause de problèmes méthodologiques, comme la petite taille de l'échantillon et la détermination de cas manquant d'uniformité, le lien entre l'exposition au mercure et le TSA demeure flou $^{38}$. Selon une méta-analyse, il se peut en outre que l'analyse du mercure 
TABLEAU 1

Sélection finale de 315 articles de recherche portant sur les liens entre facteurs

environnementaux et trouble du spectre de l'autisme, selon la dimension et le type d'article

\begin{tabular}{lcc} 
Dimension & $\begin{array}{c}\text { N }{ }^{\text {bre }} \\
\text { d'études }\end{array}$ & $\begin{array}{c}\text { N } \\
\text { reve de } \\
\text { revues de la } \\
\text { littérature }\end{array}$ \\
\hline Chimique & 94 & 44 \\
\hline Physiologique & $125^{\mathrm{a}}$ & 36 \\
\hline Nutritionnelle & 23 & 10 \\
\hline Sociale & 25 & 7 \\
\hline Autre & 4 & 2 \\
\hline
\end{tabular}

Remarque : Des études ou revues de la littérature portant sur plus d'un thème et d'autres études comportant aussi un volet méta-analyse, les totaux n'équivalent pas à 315 .

a 124 études uniques. Deux études ont utilisé les mêmes données.

dans les cheveux ne soit pas fiable ${ }^{39}$. L'utilisation des concentrations de mercure dans le sang ou les cheveux comme mesure exacte de l'exposition prénatale et au début de la vie est douteuse ${ }^{38}$ compte tenu de la demi-vie relativement courte du mercure ${ }^{40,41}$.

\section{Autres métaux lourds}

Seize études ont porté sur le lien entre l'exposition à d'autres métaux lourds et le TSA ou l'autisme en mesurant les concentrations dans des échantillons principalement de cheveux. Le plomb, le cadmium, l'aluminium et l'arsenic ont été les métaux lourds les plus étudiés chez les enfants vivant avec le TSA ou l'autisme, mais les études ont produit des résultats contradictoires (tableau 3). La plupart des études n’ont révélé aucun lien important entre l'aluminium et le TSA. Aucune des études en cause n'a porté sur les sources possibles d'exposition.

\section{Polluants}

Quelques études seulement ont porté sur le lien entre le contenu de l'eau potable et l'autisme ${ }^{42,43}$. Cinq études de population avec cas-témoins, réalisées pour la plupart en Californie, ont toutefois traité de l'exposition aux polluants atmosphériques tout en tenant compte de facteurs sociodémographiques. Ces études ont révélé des éléments de preuve relativement uniformes d'un lien entre les expositions prénatales, périnatales ou au début de la vie à des polluants atmosphériques causés par la circulation et le TSA ou l'autisme ${ }^{44-48}$ (tableau 4). L'exposition à la pollution atmosphérique liée à la circulation automobile peut causer des maladies respiratoires et cardiovasculaires et certains problèmes neurologiques en déclenchant un stress oxydatif $^{49-51}$, anomalies physiologiques courantes observées chez les enfants avec un TSA. On a établi un lien entre le lieu de résidence de la mère dans des secteurs où l'on a établi un lien entre le niveau d'exposition au dioxyde d'azote ${ }^{44,47}$, aux particules de moins de $2,5 \mu \mathrm{m}^{44,47}$ et à l'oxyde nitrique ${ }^{44}$ au cours de la gestation ou du début de la vie de l'enfant et la présence d'autisme chez un enfant. On a établi un lien aussi entre le fait d'habiter à proximité d'une autoroute au cours de la grossesse, en particulier au cours du troisième trimestre, et l'autisme chez les enfants ${ }^{46}$. Les conclusions portant sur l'exposition prénatale à d'autres polluants, dont l'ozone troposphérique et les particules de moins de $10 \mu \mathrm{m}^{44,47}$, ou l'exposition prénatale ou périnatale à des solvants et des métaux chlorés en particulier ${ }^{45,48}$ en

FIGURE 2

Sélection finale d'articles ${ }^{\mathrm{a}}$ sur les liens entre facteurs environnementaux et trouble du spectre de l'autisme, selon le sujet et le type d'article

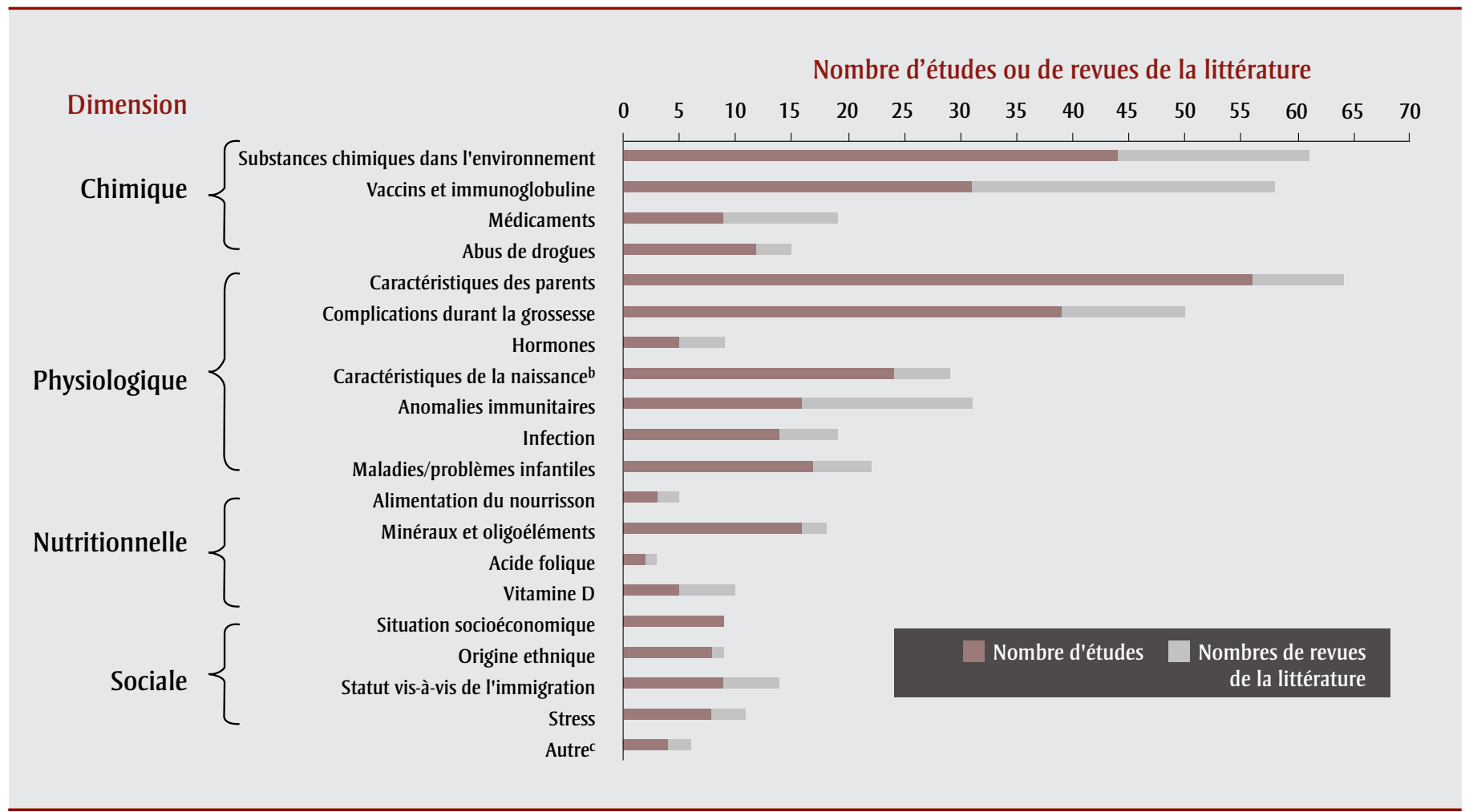

\footnotetext{
${ }^{a}$ Inclut un article qui constituait une étude de recherche primaire et une méta-analyse.

b Inclut l'ordre de naissance ou la parité, l'espacement des naissances, la saisonnalité et les naissances multiples.

c Inclut l'échographie prénatale, les rayonnements électromagnétiques et les grappes géographiques.
} 
FIGURE 3

Sélection finale d'articles sur les liens entre facteurs environnementaux et trouble du spectre de l'autisme, selon le sujet et la période, 2003 à 2008 et 2009 à 2013

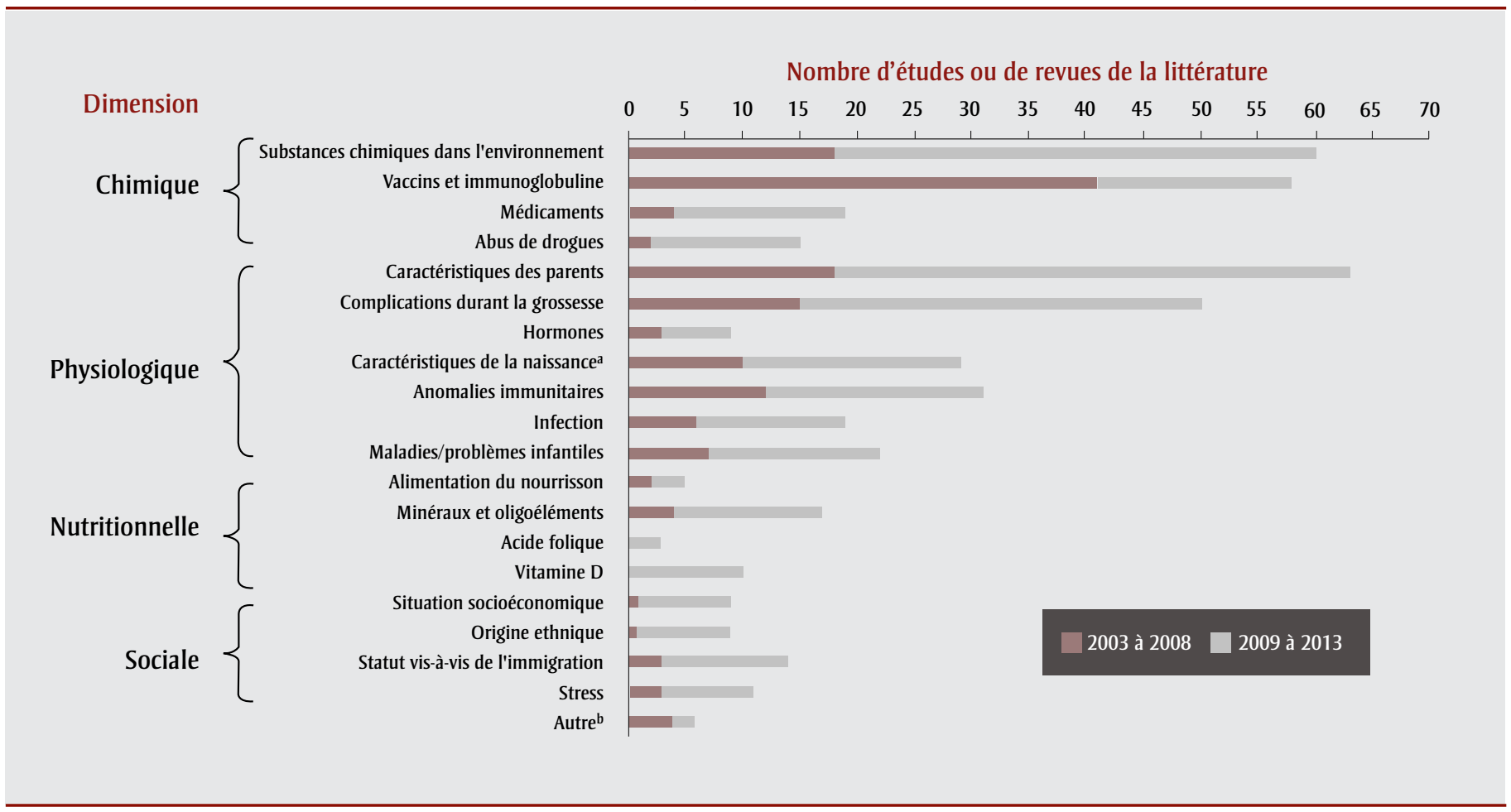

a Inclut l'ordre de la naissance et la parité, l'espacement des naissances, la saisonnalité et les naissances multiples.

b Inclut l'échographie prénatale, les rayonnements électromagnétiques et les grappes géographiques.

lien avec l'autisme ou le TSA manquent jusqu'à maintenant d'uniformité.

\section{Vaccins et médicaments}

\section{Vaccins}

Vaccin contre la rougeole, les oreillons et la rubéole (ROR)

Depuis la publication de l'étude sur la série de cas de Wakefield ${ }^{52}$, qui a révélé une prévalence accrue d'une nouvelle variante d'autisme caractérisée par des troubles gastro-intestinaux et une régression du développement, l'innocuité du vaccin ROR a été remise en question, même si l'étude en cause a été retirée par la suite à cause de fausses données. Neuf études castémoins, deux études de séries chronologiques ${ }^{53-63}$ et deux revues systématiques de la littérature ${ }^{64,65}$ n'ont révélé aucun lien important entre le vaccin ROR et le TSA ou l'autisme, même si une autre étude de séries chronologiques en a révélé un ${ }^{66}$. Un examen systématique a révélé que les études comportant le biais le plus faible basé sur les critères de qualité de l'étude n'ont pas permis d'établir l'existence d'un lien de cause à effet ${ }^{64}$. Il n'y avait en outre aucun élément de preuve portant sur un lien entre la nouvelle variante de $\mathrm{TSA}^{58,59,65}$ et le vaccin ROR, pas plus qu'entre la rougeole et l'autisme ${ }^{61,67}$.

\section{Vaccins contenant du thimérosal et immunoglobulines}

De nombreuses recherches ont porté sur le thimérosal, agent de conservation contenant $50 \%$ d'éthylmercure et utilisé dans des vaccins à flacons multiples comme le vaccin contre la diphtérie, le tétanos et la coqueluche, à cause de préoccupations soulevées par la surexposition au mercure découlant de l'expansion des calendriers de vaccination infantile survenue au cours des dernières décennies. D'autres recherches ont porté sur le thimérosal contenu dans les immunoglobulines $\mathrm{Rh}$ administrées aux femmes enceintes qui ont des problèmes d'incompatibilité Rh comme source d'exposition prénatale au mercure.

\section{Exposition postnatale au thimérosal : vaccins au cours de la petite enfance}

Sept études n’ont démontré aucun lien important entre les vaccins infantiles contenant du thimérosal et le TSA ${ }^{68-74}$, tandis que quatre en ont démontré un ${ }^{66,75-77}$. La plupart des études qui ont étayé l'existence d'un lien, toutes réalisées par les mêmes auteurs, n'ont toutefois pas tenu compte de facteurs confusionnels potentiels comme l'âge et le sexe de l'enfant, contrairement à la plupart des études n'ayant révélé l'existence d'aucun lien. Les deux études de cohorte qui ont constaté l'existence de liens positifs importants ont toutes deux utilisé une base de données sur le système de déclaration des effets secondaires et ont soulevé des critiques parce qu'elles pourraient être biaisées et ne pas être fiables, étant donné que n'importe qui peut déclarer un effet après avoir reçu un vaccin et que les diagnostics n'ont pas été validés sur le plan médical ${ }^{78}$. De plus, on n'a trouvé aucun lien entre les marqueurs auto-immuns et l'autisme chez les enfants ayant reçu des vaccins contenant du thimérosal ${ }^{79,80}$.

\section{Exposition prénatale au thimérosal : immunoglobulines Rh et anti-D}

Certaines études, pour la plupart des études cas-témoins de plus grande envergure, ont réfuté l'existence d'un lien entre l'exposition prénatale de la mère à des immunoglobuline $\mathrm{Rh}$ contenant du thimérosal et le TSA après contrôle des 
TABLEAU 2

Facteurs chimiques étudiés entre 2003 et 2013 dans le contexte du trouble du spectre de l'autisme, selon le type d'article et le sous-thème

\begin{tabular}{|c|c|c|}
\hline Sous-thème & N bre d'études & $\begin{array}{l}N^{\text {bre }} \text { de revues } \\
\text { de la littérature }\end{array}$ \\
\hline Substances chimiques dans l'environnement & & $1^{\mathrm{a}}$ \\
\hline \multicolumn{3}{|l|}{ Métaux lourds } \\
\hline Mercure & $31^{\mathrm{b}}$ & $13^{\mathrm{c}}$ \\
\hline Autres métaux lourds & 15 & 6 \\
\hline \multicolumn{3}{|l|}{ Polluants } \\
\hline Air & 5 & 1 \\
\hline Eau & 2 & 0 \\
\hline $\begin{array}{l}\text { Substances chimiques en } \\
\text { contexte professionnel }\end{array}$ & 2 & 0 \\
\hline Produits antiparasitaires & 2 & 3 \\
\hline \multicolumn{3}{|l|}{ Vaccins et médicaments } \\
\hline Vaccin ROR & $13^{\mathrm{d}}$ & 19 \\
\hline Vaccins contenant du thimérosal/lg & $17^{\mathrm{e}}$ & 16 \\
\hline Autres facteurs liés aux vaccins ${ }^{f}$ & 2 & 0 \\
\hline Valproate & 1 & 4 \\
\hline Antibiotiques & 3 & 1 \\
\hline Acétaminophène & 2 & 4 \\
\hline Terbutaline & 1 & 0 \\
\hline Antidépresseurs & 2 & 2 \\
\hline \multicolumn{3}{|l|}{ Abus de drogues } \\
\hline Alcool & 3 & 0 \\
\hline Fumée de tabac & 10 & 3 \\
\hline
\end{tabular}

Abréviations : Iǵ, immunoglobuline; ROR, rougeole, oreillons, rubéole; TSA, trouble du spectre de l’autisme.

Remarque : Certains articles de recherche pouvant être fondés sur de multiples concepts et des études ayant porté sur de multiples expositions, il peut $y$ avoir chevauchement entre les sous-thèmes.

${ }^{a}$ La revue de la littérature porte sur l'ensemble des substances chimiques dans l'environnement et n'établit pas de distinction entre différents types.

${ }^{\mathrm{b}}$ Exclut deux études qui ont réanalysé des données tirées de recherches publiées antérieurement.

c Une revue systématique de la littérature a examiné l'exposition au mercure en général, et a inclus finalement des études sur l'exposition au thimérosal.

${ }^{d}$ Deux études ont évalué le lien avec une infection par le virus de la rougeole.

e Quatre études sur l'exposition prénatale au thimérosal causée par l'immunoglobuline Rh,12 études portant sur l'exposition postnatale au thimérosal par des vaccins pour enfants et une étude portant sur l'exposition prénatale et postnatale au thimérosal.

f Études sur l'exposition à l'adjuvant d'aluminium, la fièvre suivant le vaccin et maladie mitochondriale, et les effets indésirables suivant la vaccination.

caractéristiques de la mère ou de la naissance ${ }^{37,69,81,82}$, tandis que d'autres ont trouvé des liens importants, mais sans contrôle de facteurs confusionnels possibles ${ }^{83,84}$.

\section{Médicaments}

Le recours croissant aux antidépresseurs, aux antibiotiques ${ }^{85}$ et à l'acétaminophène ${ }^{86}$ est à l'origine d'hypothèses au sujet de liens potentiels entre l'usage de ces médicaments et la montée simultanée de la prévalence du TSA au cours de la décennie 1980.

On effectue depuis peu des recherches sur le lien entre les inhibiteurs spécifiques du recaptage de la sérotonine (ISRS, une catégorie d'antidépresseurs) et le TSA, et des analyses vont dans le sens d'éléments de preuve biologiquement plausibles découlant d'un lien fondé sur des études réalisées sur des animaux et au stade préclinique $^{87,88}$. Deux études cas-témoins ont révélé un lien modéré entre l'usage prénatal d'ISRS et le TSA ${ }^{89,90}$. S'il y avait un lien de cause à effet, l'utilisation prénatale d'antidépresseurs expliquerait toutefois moins de $1 \%$ des cas de TSA ${ }^{90}$.

Trois revues systématiques de la littérature ont cité plusieurs études cliniques ayant produit des éléments de preuve d'une prévalence accrue de TSA chez les enfants exposés à l'acide valproïque (ou au valproate), anti-épileptique tératogène à spectre large et normothymique, au début de la grossesse, par rapport aux enfants non exposés ${ }^{91-93}$. Une étude de cas prospective d'envergure a en outre démontré l'existence d'un lien entre l'exposition prénatale au valproate et un risque absolu de TSA de $4,42 \%$ sur une période de 14 ans ${ }^{94}$.

Des études ont laissé entendre qu'il pouvait exister un lien entre l'usage d'antibiotiques au stade prénatal ou au début de la vie et l'autisme ${ }^{85,95,96}$. Des études ont constaté l'existence de liens contextuels entre l'usage prénatal d'acétaminophène ${ }^{97}$ et le taux de circoncision (substitut de l'acétaminophène, utilisé généralement à la suite d'une circoncision ${ }^{97}$ et d'un lien avec l'usage de l'acétaminophène après l'administration d'un vaccin ROR $^{98}$. On a laissé entendre qu'il existait des mécanismes biologiquement plausibles liés au système endocannabinoïde qui auraient un effet sur le développement du système nerveux central (SNC) ${ }^{98,99}$ ou sur une déficience de la capacité de détoxification ${ }^{86}$ à la suite d'une surdose.

\section{Usage de drogues}

\section{Fumée de tabac}

Une méta-analyse n'a fait état d'aucun lien important entre l'usage du tabac au cours de la grossesse et le TSA $^{100}$. De même, des études ultérieures n’ont révélé l'existence d'aucun lien ${ }^{101-105}$. On a toutefois démontré 
TABLEAU 3

Existence de liens entre la présence de métaux lourds chez les enfants avec trouble du spectre de l'autisme ou autisme, selon des études ou des revues de la littérature publiées entre 2003 et 2013

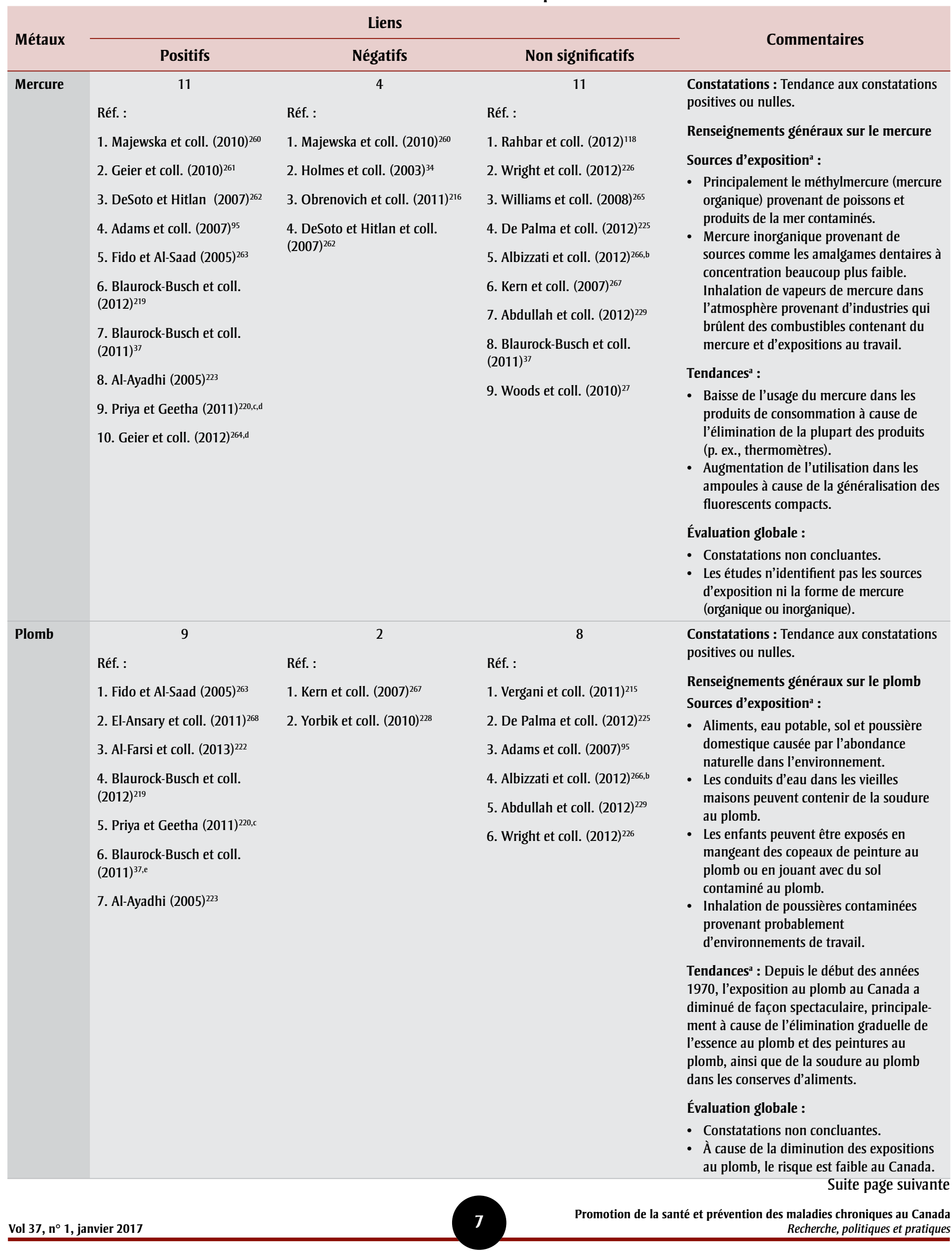


TABLEAU 3 (suite)

Existence de liens entre la présence de métaux lourds chez les enfants avec trouble du spectre de l'autisme ou autisme, selon des études ou des revues de la littérature publiées entre 2003 et 2013

\begin{tabular}{|c|c|c|c|c|}
\hline \multirow{2}{*}{ Métaux } & \multicolumn{3}{|c|}{ Liens } & \multirow{2}{*}{ Commentaires } \\
\hline & Positifs & Négatifs & Non significatifs & \\
\hline Cadmium & $\begin{array}{l}\text { Réf. : } \\
\text { 1. Vergani et coll. }(2011)^{215} \\
\text { 2. Al-Farsi et coll. }(2013)^{222} \\
\text { 3. Blaurock-Busch et coll. } \\
(2012)^{219} \\
\text { 4. Blaurock-Busch et coll. } \\
(2011)^{37} \\
\text { 5. Al-Ayadhi }(2005)^{223}\end{array}$ & $\begin{array}{l}\text { Réf. : } \\
\text { 1. Kern et coll. }(2007)^{267} \\
\text { 2. Yorbik et coll. }(2010)^{228}\end{array}$ & $\begin{array}{l}\text { Réf. : } \\
\text { 1. Albizzati et coll. }(2012)^{266, b} \\
\text { 2. Fido et Al-Saad (2005) }{ }^{263} \\
\text { 3. De Palma et coll. }(2012)^{225} \\
\text { 4. Blaurock-Busch et coll. } \\
(2011)^{37} \\
\text { 5. Wright et coll. }(2012)^{226}\end{array}$ & $\begin{array}{l}\text { Constatations : Tendance aux constatations } \\
\text { positives ou nulles. } \\
\text { Renseignements généraux sur le cadmium } \\
\text { Sources d'expositiona : } \\
\text { - Surtout aliments (p. ex., légumes à } \\
\text { feuilles, grains) et sources profession- } \\
\text { nelles. L'exposition au cadmium est faible } \\
\text { dans tous les aliments. } \\
\text { - Eau potable et produits de consommation } \\
\text { (piles, plastiques) en faible quantité. } \\
\text { - La fumée de cigarette constitue une } \\
\text { source importante chez les fumeurs. } \\
\text { Évaluation globale : } \\
\text { - Constatations non concluantes. } \\
\text { - Les fumeurs risquent davantage d'être } \\
\text { - exposés. } \\
\text { On associe de fortes concentrations de } \\
\text { cadmium inorganique au cancer du } \\
\text { poumon, à l'irritation gastro-intestinale } \\
\text { grave, ainsi qu'à des problèmes rénaux. } \\
\text { - Les études n'identifient pas les sources } \\
\text { d'exposition ni la forme } \\
\text { de cadmium. }\end{array}$ \\
\hline Aluminium & $\begin{array}{l}\text { Réf. : } \\
\text { 1. Blaurock-Busch et coll. } \\
(2011)^{37} \\
\text { 2. Blaurock-Busch et coll. } \\
(2012)^{219} \\
\text { 3. Al-Farsi et coll. }(2013)^{222}\end{array}$ & 0 & $\begin{array}{l}\quad 8 \\
\text { Réf. : } \\
\text { 1. Blaurock-Busch et coll. } \\
(2011)^{37} \\
\text { 2. Fido et coll. }(2005)^{263} \\
\text { 3. Albizzati et coll. }(2012)^{266, \mathrm{~b}} \\
\text { 4. Al-Ayadhi et coll. }(2005)^{223} \\
\text { 5. Vergani et coll. }(2011)^{215} \\
\text { 6. De Palma et coll. }(2012)^{225}\end{array}$ & $\begin{array}{l}\text { Constatations : Aucun lien important avec } \\
\text { l'aluminium dans la plupart des études. } \\
\text { Renseignements généraux sur l'aluminium } \\
\text { Sources d'exposition : } \\
\text { - Provient principalement des aliments et } \\
\text { de l'absorption orale de médicaments } \\
\text { contenant de l'aluminium (p. ex., } \\
\text { antiacides). L'adulte moyen consomme de } \\
7 \text { à } 9 \text { mg d'aluminium par jour dans son } \\
\text { alimentation. } \\
\text { - L'inhalation et le contact dermique ont } \\
\text { légèrement contribué à l'exposition } \\
\text { quotidienne. } \\
\text { - Exposition à de faibles quantités } \\
\text { d'aluminium provenant de vaccins. } \\
\text { - L'exposition importante à l'aluminium } \\
\text { (provenant probablement de sources au } \\
\text { travail) peut causer des problèmes } \\
\text { respiratoires et neurologiques. } \\
\text { Évaluation globale : Il n'y a probablement } \\
\text { aucun lien entre l'aluminium et le TSA. }\end{array}$ \\
\hline
\end{tabular}

Suite page suivante

l'existence d'un lien entre l'usage du tabac par la mère au cours de la grossesse et certaines sous-catégories de TSA, comme le syndrome d'Asperger ou le TED-NS ${ }^{106-108}$. Deux études cas-témoins d'envergure modeste ${ }^{37,109}$ indiquent qu'il peut y avoir un lien entre l'exposition de la mère à la fumée de tabac ambiante et le TSA ou l'autisme chez les enfants.

\section{Alcool}

Deux études, dont une étude de cohorte prospective, n'ont trouvé aucun lien entre la consommation prénatale d'alcool et le TSA ou l'autisme ${ }^{106110}$, tandis qu'un modeste échantillon clinique ${ }^{111}$ a démontré l'existence d'un lien important avec une consommation prénatale importante.

\section{Dimension physiologique}

La dimension physiologique regroupe de nombreux thèmes liés à la physiologie des parents et de l'enfant avec un TSA. Les caractéristiques des parents, en particulier leur âge, et les complications de la grossesse, spécialement le faible poids à la naissance et la prématurité, constituent 
TABLEAU 3 (suite)

Existence de liens entre la présence de métaux lourds chez les enfants avec trouble du spectre de l'autisme ou autisme, selon des études ou des revues de la littérature publiées entre 2003 et 2013

\begin{tabular}{|c|c|c|c|c|}
\hline \multirow{2}{*}{ Métaux } & \multicolumn{3}{|c|}{ Liens } & \multirow{2}{*}{ Commentaires } \\
\hline & Positifs & Négatifs & Non significatifs & \\
\hline Arsenic & $\begin{array}{l}\text { Réf. : } \\
\text { 1. Al-Ayadhi }(2005)^{223} \\
\text { 2. Blaurock-Busch et coll. } \\
(2011)^{37} \\
\text { 3. Blaurock-Busch et coll. } \\
(2012)^{219} \\
\text { 4. Obrenovich et coll. }(2011)^{216} \\
\text { 5. Vergani et coll. }(2011)^{215}\end{array}$ & \begin{tabular}{l}
\multicolumn{1}{c}{1} \\
Réf. : \\
1. Kern et coll. $(2007)^{267}$
\end{tabular} & $\begin{array}{l}\text { Réf. : } \\
\text { 1. Blaurock-Busch et coll. } \\
(2011)^{37} \\
\text { 2. Fido et Al-Saad }(2005)^{263} \\
\text { 3. De Palma et coll. }(2012)^{225}\end{array}$ & $\begin{array}{l}\text { Constatations : La plupart des échantillons } \\
\text { à l'étude ont révélé un lien positif même s'il } \\
\text { existe des incohérences. } \\
\text { Renseignements généraux sur l'arsenic } \\
\text { Sources d'expositione : } \\
\text { - Principalement des aliments (p. ex., } \\
\text { viande et poisson), mais provient aussi de } \\
\text { l'eau potable, du sol, de l'air et de la } \\
\text { poussière domestique, même si } \\
\text { l'exposition est habituellement faible. } \\
\text { - L'eau potable peut constituer une source } \\
\text { importante pour les populations vivant à } \\
\text { proximité d'une source d'arsenic. } \\
\text { - Présence d'arsenic dans les cigarettes, } \\
\text { même si l'exposition est mineure. } \\
\text { - Exposition importante habituellement au } \\
\text { travail, à proximité de sites de déchets } \\
\text { dangereux ou de secteurs contenant des } \\
\text { concentrations naturelles élevées. } \\
\text { Évaluation globale : } \\
\text { - Constatations non concluantes. } \\
\text { - L'exposition à l'arsenic inorganique à } \\
\text { forte concentration constitue un } \\
\text { problème de santé. L'exposition de longue } \\
\text { durée peut être cancérogène. } \\
\text { - La forme d'arsenic détectée dans le cadre } \\
\text { de ces études est inconnue. }\end{array}$ \\
\hline
\end{tabular}

Abréviation : réf., références.

Remarque : Les études peuvent analyser des liens avec différents métaux lourds ou spécimens.

${ }^{a}$ Réf. : Santé Canada ${ }^{269,270}$ et Agency for Toxic Substances and Disease Registry ${ }^{40,271-273}$.

${ }^{\mathrm{b}}$ Les analyses de sang, d'urine et de cheveux ont produit les mêmes résultats.

' Les analyses de cheveux et d'ongles ont produit les mêmes résultats.

${ }^{\mathrm{d}}$ Indique un lien avec la gravité du TSA.

${ }^{\mathrm{e}}$ Les analyses d'urine et de cheveux ont produit les mêmes résultats.

les facteurs de TSA les plus étudiés (tableau 5).

\section{Caractéristiques des parents}

\section{Âge des parents}

Cinq revues systématiques de la littérature ont fait état d'un lien entre l'âge avancé des parents et le TSA ${ }^{13,100,112,114}$. Une métaanalyse réalisée en 2011 a calculé un coefficient de probabilité (CP) regroupé d'autisme de 1,78 (intervalle de confiance [IC] à $95 \%: 1,52$ à 2,07) chez les pères âgés de 40 à 49 ans comparativement aux pères âgés de 29 ans ou moins ${ }^{113}$. Depuis la revue systématique de la littérature la plus récente $^{13}$, quatre études ont constaté l'existence d'un lien important avec le TSA ou l'autisme ${ }^{115-118}$, tandis qu'une étude n'en a trouvé aucun ${ }^{119}$. Des mutations de novo et des variations du nombre de copies du génome ont été mises en cause comme mécanisme sous-jacent possible, parce qu'elles augmentent en fonction de l'âge des parents ${ }^{120,121}$. On n'a trouvé aucun lien entre les hommes présentant des caractéristiques semblables à l'autisme et le fait de devenir parent plus tard ${ }^{122,123}$.

Une méta-analyse réalisée en 2012 a calculé que le risque relatif (RR) de TSA s'établissait à 1,31 (IC à $95 \%$ : 1,19 à $1,45)^{114}$ chez les mères de 35 ans ou plus par rapport à celles de 25 à 29 ans. Depuis la revue systématique de la littérature la plus récente ${ }^{114}$, quatre études ont constaté l'existence de liens importants entre l'âge avancé de la mère et le TSA ${ }^{117,118,124,125}$ et une autre n'en a trouvé aucun ${ }^{106}$.

Des études ont révélé que l'âge du père et celui de la mère constituaient des facteurs de risque indépendants de TSA une fois qu'on a tenu compte de l'âge du conjoint ${ }^{126-128}$ et n'ont trouvé aucun élément de preuve d'un effet synergique ${ }^{117}$. D'autres études ont tenu compte de la multicolinéarité de l'âge de la mère et de celui du père et constaté que l'âge avancé de la mère constituait le principal facteur indépendant qui contribuait à l'effet de l'âge des parents ${ }^{118,129,130}$. 
TABLEAU 4

Existence de liens entre l'exposition prénatale, périnatale et postnatale aux polluants atmosphériques chez les enfants avec un trouble du spectre de l'autisme ou l'autisme, selon des études publiées entre 2003 et 2013

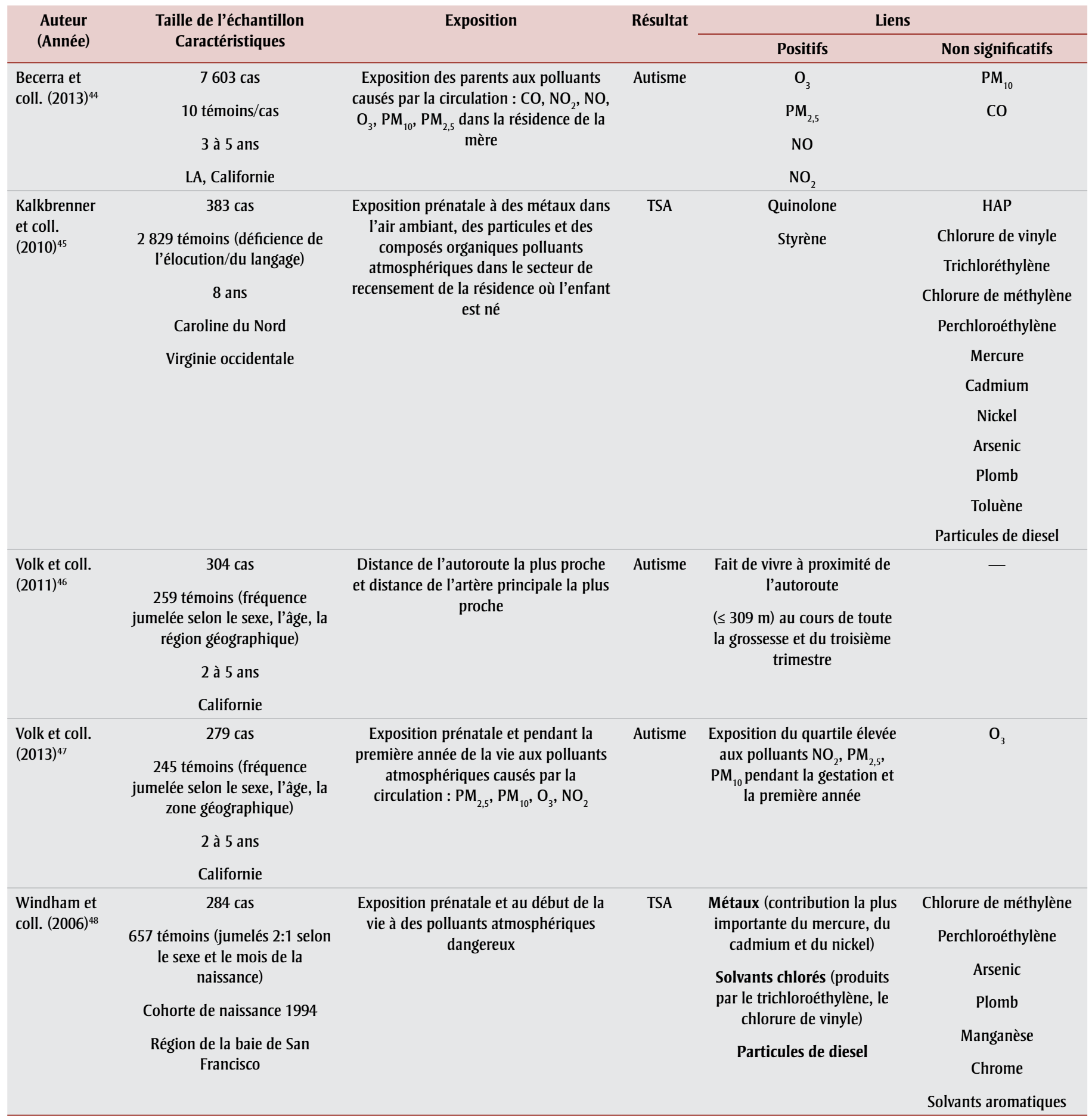

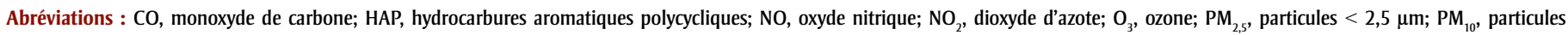
$<10 \mu \mathrm{m} ; \mathrm{TSA}$, trouble du spectre de l'autisme.

\section{Procréation assistée}

La procréation assistée et le TSA ont des facteurs de risque communs, comme un âge avancé des parents, un taux élevé de prématurité et un faible poids à la naissance $^{131}$. Une revue systématique de la littérature a cité des limites méthodologiques comme cause du manque d'uniformité des constatations sur le lien entre la procréation assistée et le TSA ${ }^{132}$. Depuis, deux études ont fait état d'un lien positif entre le recours à la procréation médicalement assistée et le $\mathrm{TSA}^{133,134}$, tandis que cinq autres n'en ont trouvé aucun ${ }^{131,135-138}$. On a toutefois constaté l'existence de liens positifs importants entre certains sous-groupes, comme les femmes de plus de 34 ans $^{136}$, les naissances multiples ${ }^{137}$ et les sujets exposés 
TABLEAU 5

Facteurs physiologiques étudiés entre 2003 et 2013 dans le contexte d'un trouble du spectre de l'autisme, selon le type d'article et le sous-thème

\begin{tabular}{|c|c|c|}
\hline Sous-thème & $N^{\text {bre }}$ d'études & $\begin{array}{l}\mathbf{N}^{\text {bre }} \text { de revues de } \\
\text { la littérature }\end{array}$ \\
\hline \multicolumn{3}{|l|}{ Caractéristiques des parents } \\
\hline Âge des parents & 39 & 7 \\
\hline Procréation assistée & 8 & 1 \\
\hline Problèmes chroniques de la mère : & 16 & 2 \\
\hline Agrégés & 3 & 0 \\
\hline Diabète $^{\mathrm{a}}$ & 10 & 2 \\
\hline Poids/prise de poids pendant la grossesse & 4 & 0 \\
\hline \multicolumn{3}{|l|}{ Complications durant la grossesse } \\
\hline Faible poids à la naissance & 16 & 6 \\
\hline Faible âge gestationnel & 19 & 6 \\
\hline Naissance postmaturée & 2 & 1 \\
\hline Enfant petit ou gros pour l'âge gestationnel & 6 & 2 \\
\hline Croissance du foetus $^{\mathrm{b}}$ & 2 & 0 \\
\hline Grappe de complications & 12 & 6 \\
\hline Toutes les complications & 39 & 11 \\
\hline \multicolumn{3}{|l|}{ Hormones } \\
\hline Testostérone & 2 & 1 \\
\hline Thyroïde & 3 & 3 \\
\hline \multicolumn{3}{|l|}{ Caractéristiques de la naissance } \\
\hline Ordre de la naissance et espacement des naissances & 15 & 4 \\
\hline Naissances multiples & 3 & 1 \\
\hline Saisonnalité des naissances & 7 & 2 \\
\hline Anomalies immunitaires & & $1^{c}$ \\
\hline Maladie auto-immune & 10 & 8 \\
\hline Inflammation du cerveau & 5 & 10 \\
\hline Autre & 1 & 0 \\
\hline Infection & 14 & 5 \\
\hline \multicolumn{3}{|l|}{ Maladies et problèmes infantiles connexes } \\
\hline Jaunisse néonatale & 6 & 3 \\
\hline Troubles épileptiques & 5 & 2 \\
\hline Stress oxydatif & 6 & 2 \\
\hline
\end{tabular}

Abréviation : AG, âge gestationnel.

Remarque : Il y a chevauchement de sous-thèmes entre études.

a Diabète préalable à la grossesse ou gestationnel.

${ }^{\text {b }}$ Mesure composite du poids à la naissance et de l'AG; circonférence de la tête dans les cas de faible poids à la naissance et enfants AG.

‘ La revue de la littérature n'établit pas de distinction entre les diverses anomalies immunitaires. à des méthodes spécifiques de procréation assistée ${ }^{131,135}$.

Maladies et problèmes chroniques de la mère

Il existe des éléments de preuve d'un lien entre certains problèmes chroniques de la mère comme la prise de poids, l'obésité et le diabète, d'une part, et le TSA chez les enfants, de l'autre. Deux ${ }^{109,139}$ études sur quatre ont fait état d'un lien positif entre des problèmes chroniques de la mère (mesure agrégée) et le TSA ou l'autisme $e^{106,109,139,140}$. Deux ${ }^{141,142}$ études de cohorte sur trois ${ }^{141-143}$ ont fait état d'un lien important entre le poids avant la grossesse, la prise de poids au cours de la grossesse et l'obésité au début de la vie, d'une part, et le TSA, de l'autre. Une méta-analyse réalisée en 2009 a fait état d'un lien positif entre le diabète gravidique de la mère et le $\mathrm{TSA}^{100}$. Depuis, les conclusions se contredisent ${ }^{102,124,139,141,144,145}$, même si certaines de ces études n'ont pas établi de distinction entre le diabète gravidique et le diabète antérieur à la grossesse.

\section{Hormones}

Testostérone prénatale

Une revue de la littérature de trois métaanalyses $^{146}$ et une étude cas-témoins ${ }^{147}$ ont cité un lien entre l'élévation des concentrations de testostérone avant la naissance et le TSA ou l'autisme. Une étude de cohorte $^{148}$ n'a toutefois découvert aucune corrélation importante avec les concentrations néonatales de testostérone.

\section{Hormones thyroïdiennes}

La dysfonction de la thyroïde, qu'elle soit attribuable à l'exposition avant la naissance ou au début de la vie, aux effets antithyroïdiens des métaux lourds, à des produits chimiques perturbateurs du système endocrinien ou à des carences alimentaires, peut avoir un effet sur le développement neurologique ${ }^{149-151}$. Les éléments de preuve sur le lien entre les concentrations néonatales ou maternelles d'hormone thyroïdienne et le TSA sont toutefois limités et manquent d'uniformité ${ }^{152-154}$.

\section{Complications au cours de la grossesse}

Une revue systématique de la littérature réalisée en 2012 a fait état d'un lien important entre les complications prénatales et néonatales de la grossesse et le $\mathrm{TSA}^{13}$. Les faits n'étaient toutefois pas concluants dans le cas de l'éclampsie ou de l'hypertension induite par la grossesse. Les constatations de plusieurs autres études ${ }^{102,103,124,125,145,155,156}$ manquaient aussi d'uniformité. 


\section{Poids à la naissance et âgé gestationnel}

Deux revues systématiques de la littérature $^{112,157}$ ont constaté que l'insuffisance de poids à la naissance constituait un important facteur de risque de TSA et, dans une d'elles (une méta-analyse), on a constaté un risque relatif (RR) de 1,63 (IC à $95 \%$ : $1,19$ à 2,33$)^{157}$. Une revue systématique de la littérature plus récente ${ }^{13}$ a toutefois produit des constatations mitigées. Depuis, trois $^{158-160}$ études sur cinq ${ }^{106,109,158-160}$ ont fait état d'un lien important entre le faible poids à la naissance et le TSA. Dans la plupart des études, le seuil de l'insuffisance de poids à la naissance s'établissait à $2500 \mathrm{~g}$.

Deux ${ }^{13,112}$ revues systématiques de la littérature sur trois ${ }^{13,112,157}$ ont étayé l'existence du lien entre un âge gestationnel peu avancé et le TSA. Depuis la revue systématique de la littérature de $2012^{13}$, la plupart des études ont produit davantage d'éléments de preuve sur ce lien ${ }^{145,159,161-163}$, même si deux études ${ }^{106,125}$ n'en ont produit aucun. Les estimations de l'effet sur le TSA ou l'autisme ont varié de 1,4 à 4,7 en fonction des études positives et de la revue systématique de la littérature ${ }^{13}$. Beaucoup d'études ont utilisé le seuil de 37 semaines pour définir les naissances "prématurées " et de 32 semaines pour définir les naissances " extrêmement prématurées ».

Des revues systématiques de la littérature $^{13,157}$ et des études subséquentes ${ }^{159,164}$ ont aussi fait état d'un lien important entre les enfants petits pour l'âge gestationnel et le TSA. D'autres études ont laissé entendre qu'il pourrait y avoir un lien entre une déviation de la croissance du fœtus ${ }^{165}$ et de son développement physique ${ }^{140}$, d'une part, et l'apparition du TSA, de l'autre.

\section{Grappe de complications de la grossesse}

Onze études, dont quatre études de cohorte, ont révélé qu'il y avait un lien important entre la mise en grappe des complications de la grossesse et le TSA ${ }^{102,104,106,109,124,141,143,166-169}$, et une seule étude cas-témoins n'en a trouvé aucun ${ }^{140}$. Quatre revues systématiques de la littérature ont aussi appuyé l'existence de ce lien ${ }^{13,100,112,157}$ et les chercheurs s'entendaient en général pour dire qu'il y avait un lien entre la présence de multiples facteurs et le TSA. Les types de complications de la grossesse analysées variaient toutefois selon les études. Beaucoup d'études ont exploré le lien entre les résultats de l'optimalité (mesures composées de la santé prénatale, périnatale et néonatale compromise dans l'ensemble) et le TSA. Des revues systématiques de la littérature ${ }^{100,157}$ ont constaté qu'une baisse de l'optimalité prénatale et néonatale est plus évidente qu'une sous-optimalité périnatale dans les cas de TSA. Depuis ces revues de la littérature, une étude de cohorte prospective a toutefois révélé qu'il y avait un lien important entre la présence d'au moins quatre facteurs de sous-optimalité obstétrique (principalement prénataux et périnataux) lors d'une première naissance et le TSA $^{124}$. Trois autres études ont constaté que les problèmes de naissance sous-optimale ${ }^{101,105,141}$ et des complications prénatales de la grossesse ${ }^{102,106,141}$ étaient plus courants chez les enfants avec le TSA que ceux sans ce trouble.

Des revues systématiques de la littérature $^{112,157}$ ont mis en cause l'hypoxie fœtale (privation intra-utérine d'oxygène) dans l'étiologie du TSA, sur la base de plusieurs facteurs périnataux pouvant servir de marqueurs de l'hypoxie, en particulier un indice d'Apgar faible, une césarienne et un retard de croissance. Une étude de cohorte en population ${ }^{170}$ a néanmoins établi un lien ténu entre l'hypoxie fœtale et le TSA.

\section{Caractéristiques de la naissance}

\section{Ordre et espacement des naissances}

Des revues systématiques de la littérature constatent que l'on est plus susceptible de diagnostiquer un TSA chez les enfants aînés que chez les enfants nés au troisième rang ou plus $\operatorname{tard}^{13,100}$. La méta-analyse a révélé que les enfants aînés présentaient un risque d'autisme plus élevé de $61 \%$ que les enfants nés au troisième rang ou plus tard ${ }^{100}$. Cette constatation concorde aussi avec certaines études plus récentes ${ }^{106,140,171,172}$, dont une a porté seulement sur des patients qui avaient le syndrome d'Asperger ${ }^{171}$. On a établi un lien entre la parité sous-optimale (plus de deux naissances) et le TSA ${ }^{124}$. Le fait d'être né plus tôt et une plus grande parité semblent constituer des facteurs de risque contradictoires, parce que les aînés des familles de deux enfants et les enfants nés plus tard dans des familles plus nombreuses sont plus susceptibles d'avoir le TSA $^{100}$. Des études ont en outre indiqué qu'il peut y avoir un lien entre la gravité des symptômes du TSA et l'ordre de naissance, même si ces études ne s'entendaient pas sur un lien plus étroit entre l'ordre des naissances et la gravité des symptômes ${ }^{173,174}$, peut-être parce que les intervalles entre les grossesses peuvent aussi constituer un facteur ${ }^{174}$. On a aussi établi un lien entre un intervalle de moins de
28 mois $^{141}$ entre les grossesses ou de moins d'un an et l'autisme chez le deuxième enfant ${ }^{175}$.

\section{Autres caractéristiques de la naissance}

On a établi un lien entre des naissances multiples et le TSA, d'après une métaanalyse $^{157}$ ainsi que d'après une étude de cohorte $^{176}$, tandis qu'une étude écologique n’a pas révélé de lien ${ }^{177}$. Une méta-analyse a aussi révélé un lien important entre les naissances estivales et le TSA ${ }^{157}$. Des faits contradictoires sont toutefois présents dans plusieurs études ${ }^{144,178-181}$.

\section{Anomalies immunitaires}

Une analyse des tendances de la recherche révèle que la dysrégulation immunitaire a fait l'objet de nombreuses études, qui ont produit les éléments de preuve les plus solides des anomalies physiologiques dans les cas de TSA ${ }^{12}$. Les premiers éléments de preuve ont indiqué l'existence d'un lien possible entre le TSA et deux types principaux de dysfonction immunitaire, soit l'autoimmunité et l'inflammation du cerveau ${ }^{182}$. On a en outre observé une réduction importante de la concentration d'immunoglobuline $\mathrm{G}$ dans le sang des nouveau-nés dans des spécimens archivés provenant de nouveau-nés chez lesquels on a diagnostiqué par la suite un TSA, par rapport aux nouveau-nés chez lesquels on n'a diagnostiqué aucun TSA par la suite ${ }^{183}$.

\section{Maladies auto-immunes}

Plusieurs études ont porté sur le rôle de l'auto-immunité dans le TSA. Trois études cas-témoins ont démontré de façon uniforme que la réactivité des anticorps aux protéines du cerveau du fœtus humain a une prévalence supérieure chez les mères d'enfants avec le TSA, ou plus précisément avec l'autisme, que chez les mères d'enfants qui n’ont pas de TSA ou plus précisément d'autisme ${ }^{184-186}$. On peut en outre constater une élévation des concentrations d'autoanticorps des récepteurs du folate sérique chez les enfants atteints d'autisme limité, avec ou sans déficit neurologique, par rapport aux sujets témoins qui ne souffrent pas d'autisme ${ }^{187}$. Ces résultats laissent entendre que le transfert du folate au fœtus au cours de la grossesse peut être bloqué, ce qui peut accroître les risques d'anomalies du tube neural ${ }^{188}$. Comme on a constaté ces concentrations élevées d'auto-anticorps aussi chez au moins un parent d'enfants atteints d'autisme, il se peut que certains parents contribuent à l'étiologie de l'autisme ${ }^{189}$. 
Deux études de cohorte et une étude castémoins ont révélé l'existence d'un lien entre les antécédents maternels ou familiaux de maladies auto-immunes et le $\mathrm{TSA}^{124,190}$ ou la régression autistique ${ }^{191}$, tandis qu'une autre étude cas-témoins n'a découvert aucun lien ${ }^{192}$. Les maladies autoimmunes spécifiques associées au TSA étaient toutefois différentes selon les études. Les auteurs de revues de la littérature ont discuté d'éléments de preuve de plus en plus nombreux en lien avec le rôle de l'auto-immunité dans le TSA, éléments tirés principalement de modèles animaux et d'études cliniques portant sur des sujets humains, mais ils ont indiqué qu'il fallait déterminer les fonctions des auto-anticorps susceptibles d'avoir une incidence sur le développement neurologique ${ }^{193-196}$.

\section{Inflammation du cerveau}

On a aussi mis en cause l'inflammation du cerveau dans l'étiologie du TSA. Selon les revues de la littérature basées principalement sur des études cliniques portant sur des animaux et des humains, des accidents immunitaires au début de la vie, comme des substances toxiques, des additifs alimentaires ou le stress, peuvent entraîner une cascade d'excitotoxicité dans le cerveau $^{197-199}$ et peuvent être liés à une dysrégulation de la neurotransmission du glutamate ${ }^{199,200}$. Cette cascade peut déclencher la production de cytokines proinflammatoires, ce qui entraîne une inflammation chronique qui a des répercussions sur le développement neurologique.

De nouveaux éléments de preuve, tirés principalement d'études cas-témoins, ont fait état d'une production accrue de cytokines pro-inflammatoires dans le sérum ou le liquide céphalorachidien d'enfants vivant avec le TSA ou dans le liquide amniotique de leur mère ${ }^{201-203}$, même si l'on a aussi observé une baisse de la concentration néonatale de cytokines ${ }^{204}$. Une altération importante de la fonction immunitaire cellulaire adaptative chez les enfants avec un TSA peut refléter une activation défectueuse du système immunitaire, qu'il est possible de relier en retour à l'incapacité attribuable au TSA ${ }^{203}$. On n'a en outre signalé aucune différence importante en matière de concentrations de certaines chimiokines dans le sang du nouveau-né et le liquide amniotique ${ }^{201,205}$, sauf dans un sousgroupes avec un diagnostic de TSA basé sur les critères de diagnostic les plus récents ${ }^{201}$.
Infection

Les infections peuvent déclencher une inflammation chronique du SNC, qui a des répercussions sur le développement et la maturation du cerveau, ce qui participe à l'étiologie du $\mathrm{TSA}^{198}$. Selon une métaanalyse $^{100}$ et une recherche documentaire effectuée en $2012^{206}$, il n'y avait toutefois aucun lien important entre cette infection chez la mère et le TSA. Une revue systématique réalisée en 2012 a indiqué que d'autres recherches sont nécessaires pour explorer ce lien ${ }^{13}$. Depuis, une seule étude cas-témoins a découvert un lien important ${ }^{106}$, tandis qu'une étude de cohorte et trois études cas-témoins n'en ont découvert aucun $^{96,125,140,207}$. La définition de l'infection chez la mère varie selon les études : elle peut englober toute infection survenue au cours de la grossesse ou seulement certaines infections précises, par exemple la grippe $^{207}$. Des études ont laissé entendre qu'il existait un lien entre de la fièvre chez la mère au cours de la grossesse ${ }^{207}$ ou des épisodes fébriles prolongés ${ }^{96}$ et le TSA, même si les faits sont mitigés ${ }^{145,208}$.

\section{Maladies infantiles associées}

\section{Jaunisse néonatale}

La jaunisse néonatale, causée par une élévation des concentrations de bilirubine sérique (hyperbilirubinémie) est préoccupante parce que la bilirubine non conjuguée peut être toxique pour le SNC en développement. Cinq études ${ }^{125,144,209-211}$ et deux revues systématiques de la littérature $^{13,157}$ ont porté sur ce facteur de risque. D’après les revues systématiques de la littérature, on a établi un lien entre l'hyperbilirubinémie et un risque plus élevé d'autisme $^{13,157}$, avec un effet sommaire estimé à 1,87 (IC à $95 \%: 1,01$ à 3,47 ) d'après la méta-analyse ${ }^{157}$.

\section{Troubles épileptiques}

Une méta-analyse n’a indiqué aucun lien important entre les crises d'épilepsie néonatales et le $\mathrm{TSA}^{157}$, mais quatre études d'envergure modeste ont produit des éléments de preuve d'un lien potentiel ${ }^{125,212-214}$.

\section{Stress oxydatif}

Selon une revue de la littérature consacrée aux tendances de la recherche, le stress oxydatif a constitué une des anomalies physiologiques les plus étudiées dans les cas de TSA, avec l'une des assises factuelles les plus solides, principalement constituée d'études cliniques et animales ${ }^{12}$. Six études cas-témoins ont indiqué que le stress oxydatif jouait un rôle chez les patients ayant un TSA ${ }^{29,30,215-218}$ compte tenu de différents biomarqueurs du stress oxydatif et de déficits des systèmes antioxydants. Les biomarqueurs indiquaient une altération des niveaux des systèmes de défense contre le stress oxydatif, dont des métallothionéines et des enzymes antioxydants $^{215}$. Les autres marqueurs du stress oxydatif associés à la toxicité des métaux lourds ou à une faible capacité de détoxification étaient les marqueurs anormaux du métabolisme des thiols ${ }^{216}$, des anomalies de la transsulfuration ${ }^{30}$, la présence d'acides organiques urinaires ${ }^{29}$ et l'élévation des concentrations des ions plomb et potassium, de l'activité de l'ATPase et des concentrations de produits de la peroxydation des lipides ${ }^{217}$.

\section{Dimension nutritionnelle}

Les carences en minéraux et les concentrations d'oligoéléments ont constitué les facteurs nutritionnels les plus étudiés dans le contexte du TSA, suivis de la carence en vitamine D (tableau 6).

\section{Minéraux et oligoéléments}

Les éléments de preuve portant sur les carences en minéraux et les concentrations d'oligoéléments chez les enfants avec un TSA ou plus spécifiquement l'autisme sont tirés principalement d'études cas-témoins au cours desquelles on a analysé des échantillons de cheveux, et ils demeurent non concluants. Les études portant sur des liens entre les concentrations de zinc ${ }^{37,95,215,219-223}$, de fer $37,215,216,219,222-224$, de magnésium ${ }^{37,219,220,222,223}$, de cuivre ${ }^{37,215,216,219,220,222,223,225,226}$, de molybdène $^{37,219,222,223,225}$, de nickel ${ }^{215,219,222,223,225}$ et de

\section{TABLEAU 6}

Facteurs nutritionnels étudiés entre 2003 et 2013 dans le contexte d'un trouble du spectre de l'autisme, selon le type d'article et le sous-thème

\begin{tabular}{|lcc|}
\hline Sous-thème & $\begin{array}{c}\text { Nbre } \\
\text { d'études }\end{array}$ & $\begin{array}{c}\text { Noves de } \\
\text { revues de } \\
\text { la } \\
\text { littérature }\end{array}$ \\
\hline $\begin{array}{l}\text { Minéraux et } \\
\text { oligoéléments }\end{array}$ & 16 & 2 \\
\hline Vitamine D & 5 & 5 \\
\hline $\begin{array}{l}\text { Alimentation du } \\
\text { nourrisson }\end{array}$ & 3 & 2 \\
\hline Acide folique & 2 & 1 \\
\hline
\end{tabular}

Remarque : Il y a un peu de chevauchement entre les sous-thèmes des études ou revues de la littérature. 
sélénium $^{37,219,220,223,225}$ et le TSA ont produit des résultats contradictoires. On a aussi documenté des carences en calcium reliées au TSA ou à l'autisme chez des enfants $^{219,222,223,227}$, mais avec un manque de cohérence $^{37,215}$. Outre ce manque d'uniformité, les différences au niveau des concentrations de cobalt ${ }^{37,215,222,223,225,226}$, de chrome $^{37,215,222,223,225,228}$ et de manganèse $\mathrm{e}^{37,215,219,223,225,226,229}$ chez les enfants avec et sans TSA ou autisme étaient pour la plupart non significatives.

\section{Vitamine D}

La vitamine $\mathrm{D}$ joue un rôle crucial dans diverses fonctions comme le développement neurologique, la réaction anti-inflammatoire et les modes de détoxification ${ }^{230,231}$. Les éléments de preuve portant sur l'association entre une carence en vitamine $\mathrm{D}$ et le TSA demeurent toutefois limités et indirects. Deux études écologiques ${ }^{63,232}$ et une étude cas-témoins ${ }^{227}$ ont fait état d'un lien entre une carence en vitamine D chez les mères ou chez leurs enfants atteints d'autisme, d'une part, et l'apparition de l'autisme, de l'autre. On a laissé entendre que la migration peut jouer un rôle dans l'étiologie du TSA parce que les taux de prévalence de l'autisme sont plus élevés chez les immigrants des pays du nord de l'Europe et parce que les immigrants à la peau foncée sont plus sensibles à la carence en vitamine $\mathrm{D}$ à cause de la pigmentation de leur peau ${ }^{233}$. Une étude cas-témoins d'envergure modeste ${ }^{234}$ et une récente étude de cohorte prospective réalisée en Australie ${ }^{235}$ n'ont toutefois constaté aucun lien important entre les concentrations de vitamine $\mathrm{D}$ dans le sérum de la mère et le TSA ou une majorité de caractéristiques analogues à l'autisme chez ses descendants. De plus, une revue systématique de la littérature a conclu qu'il n'y avait pas suffisamment d'éléments de preuve d'un lien et que d'autres études de population longitudinales s'imposent, étant donné le caractère plausible des éléments de preuve biologiques ${ }^{230}$.

\section{Alimentation des nourrissons}

Nous avons trouvé peu d'études sur les méthodes d'alimentation des nourrissons dans le contexte du TSA, même si deux études cas-témoins ont établi un lien important entre l'absence d'allaitement ou le début tardif de l'allaitement et le TSA ou l'autisme ${ }^{236,237}$, ce qui ne concorde pas avec les résultats d'une étude écologique ${ }^{63}$.

\section{Acide folique}

Les éléments de preuve à l'appui d'un lien entre l'apport en acide folique et le TSA sont très rares, même si une récente étude de cohorte prospective a révélé que l'absorption d'acide folique par la mère peut agir contre le TSA ${ }^{238}$, à l'inverse d'une étude écologique qui a découvert des corrélations positives entre le pourcentage de prescriptions de vitamines prénatales et de vitamines pédiatriques contenant de l'acide folique et l'incidence de TSA ${ }^{239}$.

\section{Dimension sociale}

La situation socioéconomique (SSE), l'origine ethnique, le statut vis-à-vis de l'immigration et le stress sont les facteurs sociaux analysés de façon récurrente dans le contexte du TSA (tableau 7).

\section{Situation socioéconomique}

Neuf études ${ }^{102,119,172,240-245}$ ont dégagé l'importance de la SSE comme prédicteur du risque de TSA, mais les constatations ont été mitigées. Des études, surtout américaines $^{172,240-242}$, ont établi un lien entre une meilleure SSE, mesurée par des substituts comme le revenu de la mère, sa profession et ses études, et le TSA. Lorsque les chercheurs de l'une de ces études ${ }^{242}$ ont procédé à une sous-analyse par détermination des cas, ils n'ont établi aucun lien avec la SSE à partir des cas provenant de sources scolaires seulement. Une étude réalisée en Australie $^{172}$ a établi une corrélation négative entre la résidence de la mère en région éloignée et des cas de TSA sans déficience intellectuelle (DI). D’autres études ont toutefois produit des résultats différents ${ }^{102,119,243,244}$. Dans des pays dotés de programmes universels de soins de santé,

\section{TABLEAU 7}

Facteurs sociaux étudiés entre 2003 et 2013 dans le contexte d'un trouble du spectre de l'autisme, selon le type d'article et le sous-thème

\begin{tabular}{lcc}
\hline Sous-thème & $\begin{array}{c}\mathbf{N}^{\text {bre }} \\
\text { d'études }^{\text {setuation }}\end{array}$ & $\begin{array}{c}\mathbf{N}^{\text {bre de }} \\
\text { revues de la } \\
\text { littérature }\end{array}$ \\
\hline $\begin{array}{l}\text { Situcioéconomique } \\
\text { Socigine ethnique }\end{array}$ & 9 & 0 \\
\hline $\begin{array}{l}\text { Statut vis-à-vis de } \\
\text { l'immigration }\end{array}$ & 9 & 1 \\
\hline Stress & 8 & 5 \\
\hline
\end{tabular}

Remarque : Il y a chevauchement entre les divers sous-thèmes d'études ou revues de la littérature. comme la Suède et le Canada, un lien a été établi entre le revenu familial ou maternel moins élevé et le TSA, ce qui indique que les liens entre une SSE élevée et le TSA constatés principalement dans des études réalisées aux États-Unis peuvent être le reflet des inégalités d'accès aux services de santé (p. ex., sous-diagnostic du TSA dans les familles dont la SSE est moins bonne) ${ }^{102,244}$. De plus, une analyse spatiale de l'incidence de l'autisme dans des régions de la Californie a révélé une incidence plus élevée, corrélée de manière indépendante aux études plus poussées faites par les parents ${ }^{245}$.

\section{Origine ethnique}

La plupart des études réalisées sur le lien entre l'origine ethnique de la mère et le TSA ont fait état d'un lien important ${ }^{102,119,172,242,243,246}$, à l'exception d'une étude réalisée au Royaume-Uni ${ }^{247}$. On a plus particulièrement établi un lien entre l'origine ethnique minoritaire de la mère, par exemple le fait d'être Autochtone, et une diminution du nombre de diagnostics de TSA chez ses descendants ${ }^{102,119,172,243}$ et calculé un $\mathrm{CP}$ variant de 0,33 à 0,83 pour certains groupes ethniques non blancs comparativement aux personnes blanches d'origine non hispanique. De plus, les enfants d'origine ethnique noire, hispanique ou " autre » étaient moins susceptibles que les enfants blancs d'avoir le TSA, qui était plus prononcé chez les enfants de certains groupes ethniques atteints de DI, selon une étude américaine ${ }^{246}$. La constatation selon laquelle les groupes minoritaires seraient moins susceptibles de recevoir un diagnostic de TSA est peut-être attribuable à un biais de détermination. L'évaluation différentielle des problèmes intellectuels et développementaux des enfants peut expliquer ces disparités entre les ethnies. En effet, une autre étude américaine a constaté un lien entre la race noire et des risques accrus de TSA, en particulier combiné avec une DI, mais la détermination des cas relevait sans doute davantage de sources scolaires $^{242}$. Ces études laissent entendre qu'il pourrait exister des disparités raciales ou ethniques au niveau du diagnostic de TSA dans certains groupes, une DI comorbide pouvant avoir des répercussions sur la détection du trouble.

\section{Statut vis-à-vis de l'immigration}

Le fait que la mère soit immigrante est un des facteurs sociaux répertorié dans huit études ${ }^{104,208,243,247-251}$ et deux revues systématiques de la littérature ${ }^{13,100}$ établissant un 
lien avec le TSA ou un sous-type de TSA. Les mères migrantes présentaient une prévalence plus élevée de descendants avec un TSA selon des études réalisées en Suède, au Royaume-Uni et en Australie. La méta-analyse a révélé un lien marginal entre une mère immigrante et l'autisme (estimation de l'effet sommaire $=1,28$; $p=0,06)^{100}$. Dans les pays nordiques, ce lien se révélait statistiquement significatif, le risque augmentant de $58 \%$ chez les enfants dont la mère était d'origine étrangère ${ }^{100}$. Selon une étude réalisée en Suède, il se pourrait que les parents migrants provenant de pays classés au bas de l'échelle selon l'indice du développement humain risquent davantage d'avoir des enfants ayant le TSA avec une DI comorbide que les parents d'origine suédoise, particulièrement lorsque la migration s'est produite au cours de la grossesse, ce qui sous-entend un lien potentiel avec le stress chez la mère ${ }^{249}$. Cependant, les parents migrants provenant de pays en développement risqueraient moins d'avoir des enfants atteints de TED-NS ou du syndrome d'Asperger ${ }^{250}$. Il a aussi été démontré que le contexte sociopolitique, en particulier les changements dans la politique d'immigration, a un effet sur les tendances du taux de diagnostic de l'autisme ${ }^{251}$. Par exemple, une baisse des taux d'autisme chez les enfants hispaniques aux ÉtatsUnis a été attribuée à une réticence des parents qui sont des immigrants sans papier à faire appel à des services de diagnostic de peur d'être signalés à la police et déportés, dans un contexte d'application de politiques anti-immigration.

\section{Stress chez la mère}

L'exposition prénatale à des facteurs de stress environnementaux, en particulier des anomalies immunologiques et neuroinflammatoires liées au stress et une dysfonction placentaire susceptibles d'avoir un effet sur le développement neurologique du fœtus, pourrait jouer un rôle dans l'étiologie du TSA ${ }^{252,253}$. Un examen systématique réalisé en 2012 a conclu qu'il fallait étudier ce lien plus à fond pour produire des éléments de preuve conclu$a_{n} s^{13}$. Depuis, une étude de cohorte et une étude cas-témoins ont fait état d'un lien entre le stress chez la mère durant la grossesse et la conception d'un enfant atteint de $\mathrm{TSA}^{106,254}$, tandis que trois études castémoins n’ont trouvé aucun lien ${ }^{140,145,255}$. Dans beaucoup de ces études, les « facteurs de stress " sont définis soit de manière générale comme tout type d'événement stressant $^{106,140,145,255}$, soit plus spécifiquement en lien avec certains événements stressants comme la violence au cours de la petite enfance ${ }^{254}$.

\section{Autres dimensions}

Outre les dimensions chimique, physiologique, nutritionnelle et sociale, plusieurs autres facteurs ont été dégagés dans les analyse de leurs liens potentiels avec le TSA. Deux études ont montré que les naissances d'enfants vivant avec l'autisme se produisent par grappes géographiques, ce qui pourrait indiquer que des facteurs locaux interviennent dans la prévalence ${ }^{247,256}$. Une autre étude a montré que même si l'hypothèse que les rayons électromagnétiques pourraient constituer un facteur de risque est plausible du point de vue biologique, il n'existe encore aucun élément de preuve épidémiologique ${ }^{257}$. D’autres études n'ont trouvé aucun lien important entre l'exposition prénatale aux ultrasons et le TSA 256,258 .

\section{Analyse}

La recherche sur la contribution de l'environnement à l'étiologie du TSA a porté surtout sur les facteurs de risque physiologiques ou chimiques, et moins sur les facteurs sociaux et nutritionnels. Dans le contexte de ces dimensions, l'importante littérature disponible est largement criblée de résultats sans cohérence. L'hétérogénéité dans les groupes d'étude, l'exposition et l'évaluation des résultats, l'évolution des critères de diagnostic ou des phénotypes du TSA est évidente, et tous ces facteurs varient entre études et sont susceptibles d'avoir une incidence sur la validité des constatations.

Les biomarqueurs de l'exposition aux métaux lourds, et en particulier au mercure, tirés des concentrations mesurées dans des spécimens biologiques comme les cheveux, le sang et l'urine, ont fait l'objet d'études intensives, mais le lien entre l'exposition et le TSA demeure incertain parce que les constatations sont contradictoires. Comme la plupart de ces études mesurent seulement les biomarqueurs des métaux lourds et ne déterminent pas les sources réelles d'exposition, on ne connaît pas la temporalité du lien. De plus, beaucoup d'études sur les biomarqueurs ont porté sur des échantillons de petite taille. Ces constatations concordent avec une revue systématique de la littérature récente (publiée en 2014, après la fin de notre étude) de Rossignol et ses collaborateurs ${ }^{11}$ portant sur le lien entre l'exposition à des substances toxiques environnementales et le TSA.

Par ailleurs, les éléments de preuve, de plus en plus nombreux, sur le lien entre les polluants atmosphériques causés par la circulation et le TSA ou l'autisme sont relativement constants, même si des recherches plus poussées s'imposent pour établir la spécificité du lien et en améliorer la validité externe au-delà du paysage américain. Ces constatations concordent aussi avec l'étude de Rossignol, qui a constaté que la pollution atmosphérique constitue le facteur de risque chimique présentant les éléments de preuve les plus solides d'un lien avec le TSA, même si le lien avec les produits antiparasitaires s'est révélé aussi relativement solide.

En dépit de nombreuses études sur le lien entre le vaccin ROR ou les vaccins contenant du thimérosal et le TSA, il n'y a pas suffisamment d'éléments de preuve convaincants à l'appui de ce lien. En outre, les éléments de preuve d'un lien entre l'exposition du fœtus ou de l'enfant à divers médicaments et le TSA sont limités à quelques études. On constate aussi un manque de lien entre l'exposition à la fumée de tabac et le TSA, même si des études ont signalé un lien potentiel avec le TED-NS, ce qui justifie une étude plus poussée des sous-groupes de TSA séparément si l'on veut clarifier des liens de cause à effet. Les études sur l'exposition aux produits chimiques dans un cadre professionnel, aux produits antiparasitaires et à l'alcool se sont révélées en outre limitées.

Les facteurs physiologiques incriminés le plus généralement et le plus souvent dans l'apparition du TSA sont l'âge avancé des parents, une insuffisance de poids à la naissance, la prématurité et une grappe de complications de la grossesse. On a démontré aussi un lien constant entre l'hyperbilirubinémie et le TSA. Compte tenu des constatations préliminaires, il faut pousser la recherche au sujet des effets de l'âge avancé des grands-parents sur le TSA ${ }^{115,259}$. Il faut de plus procéder à d'autres études pour comprendre les mécanismes des liens entre ces facteurs physiologiques et le TSA. On a aussi signalé chez les enfants vivant avec le TSA ou leur mère l'apparition 
d'éléments de preuve épidémiologiques portant sur des anomalies immunitaires liées à l'auto-immunité et à l'inflammation du cerveau. D'autres travaux s'imposent toutefois pour déterminer la temporalité du lien et préciser le rôle potentiel de ces facteurs dans l'étiologie du TSA.

Des recherches plus poussées s'imposent aussi pour comprendre d'autres facteurs physiologiques comme les caractéristiques de la naissance, les problèmes chroniques de la mère, les hormones et les problèmes infantiles liés au TSA. Des études ont révélé l'existence d'un lien potentiel entre des problèmes chroniques comme la prise de poids au cours de la grossesse ou le diabète de la mère, d'une part, et le TSA, de l'autre. À cause de l'hétérogénéité et du manque de spécificité des expositions, une recherche plus poussée est toutefois nécessaire. Il existe des éléments de preuve sur l'existence d'un lien entre l'ordre de naissance plus précoce, des naissances plus nombreuses et de brefs intervalles entre les grossesses, d'une part, et le TSA, de l'autre, même si la contribution de ces facteurs à son étiologie n'est pas claire. Quelques études épidémiologiques d'envergure modeste ont démontré un lien entre le stress oxydatif et le TSA.

Le lien entre les facteurs nutritionnels et le TSA n'a pas fait l'objet d'études minutieuses, contrairement à celles qui ont porté sur les facteurs chimiques et physiologiques. Même si plusieurs études ont analysé les liens avec des carences en minéraux ou oligoéléments et en vitamine $\mathrm{D}$, les éléments de preuve manquent de cohérence et sont indirects. Par exemple, on a utilisé la naissance à l'étranger de la mère ou son origine ethnique comme substituts de la concentration en vitamine $\mathrm{D}$ basée sur l'hypothèse, qui pourrait être inexacte, selon laquelle les femmes de ces catégories auraient une pigmentation cutanée plus foncée, ce qui aurait un effet sur leurs concentrations de vitamine $\mathrm{D}^{233}$. On pourrait trouver des éléments de preuve plus directs d'un lien entre la vitamine D et le TSA en mesurant l'exposition directe.

La littérature actuelle indique que les liens entre les facteurs sociaux comme la SSE et l'origine ethnique, d'une part, et le TSA, de l'autre, seraient variables selon les pays, en fonction des biais potentiels de détermination des cas. On a toutefois établi un lien constant avec le fait que la mère soit immigrante, et d'autres études s'imposent pour déterminer si ce lien peut être attribuable à la SSE, à l'adaptation à un nouvel environnement, au stress ou à des changements d'exposition à la vitamine $\mathrm{D}$, comme le laissent entendre certaines études.

Dans l'ensemble, le manque de cohérence, de temporalité et de spécificité des associations observées dans le cadre de nombreuses études empêche d'établir un lien de cause à effet. Des études longitudinales seraient utiles pour définir ces liens de cause à effet potentiels, en contribuant à en déterminer la temporalité. Des méthodes uniformes de mesure de l'exposition et de détermination des cas ainsi que la prise en considération des facteurs de confusion potentiels pourraient réduire l'hétérogénéité des données. Les mécanismes qui soustendent certains de ces liens doivent être étudiés dans le cadre de recherches biologiques plus poussées.

\section{Forces et limites}

Parmi les études qui ont porté sur les facteurs potentiels de risque de TSA jusqu'à maintenant, aucune n’a analysé la portée complète de la contribution de l'environnement au TSA. Nous avons procédé à une recherche documentaire systématique et détaillée, ce qui fait que même si elle n'a pas recensé tous les articles pertinents, ont été sélectionnés de nombreux facteurs potentiellement importants, qui sont susceptibles de contribuer à brosser un tableau exact de l'envergure de la contribution de l'environnement à l'étiologie du TSA.

Par ailleurs, même si la stratégie de recherche d'articles pertinents sur le TSA visait l'intégralité, nous n'avons pas effectué de recherche manuelle dans des revues scientifiques clés ni de recherche sur les citations mentionnées dans les études et la littérature grise incluse. De plus, d'autres renseignements sur les facteurs environnementaux n'ont peut-être pas été sélectionnés si les articles à leur sujet traitaient aussi de modèles animaux provenant d'études génétiques, ces dernières ayant été exclues. Ce phénomène est évident dans le cas de la recherche sur les anomalies du système immunitaire et le stress oxydatif, parce que les articles extraits étaient moins nombreux alors même que les revues de la littérature indiquaient un volume important de recherche à leur sujet, ces recherches étant probablement tirées d'études cliniques et animales. Comme notre étude de délimitation visait à évaluer l'état actuel des connaissances sur les facteurs de risque potentiels de TSA liés à l'environnement, nous n'avons pas évalué la qualité de ces études pour déterminer la solidité des éléments de preuve.

\section{Conclusion}

Notre étude de délimitation a porté sur la recherche effectuée entre 2003 et 2013 à propos des facteurs environnementaux potentiellement reliables au TSA, regroupés en quatre catégories : facteurs chimiques, physiologiques, nutritionnels et sociaux. Nous avons constaté que les facteurs physiologiques étaient en particulier un âge avancé des parents, une insuffisance de poids à la naissance, la prématurité, une hyperbilirubinémie et des grappes de complications de la grossesse, éléments toujours signalés comme facteurs de risque de TSA. Des éléments de preuve d'un lien avec des polluants atmosphériques causés par la circulation commencent à se dégager, mais les recherches sur les facteurs nutritionnels associés au TSA demeurent limitées. Parmi les facteurs de la dimension sociale, le lien entre l'immigration et le TSA a été régulièrement confirmé, ce qui justifie une recherche plus poussée. D'autres liens avec des facteurs sociaux comme la SSE et l'origine ethnique peuvent être le reflet de disparités au niveau du diagnostic de TSA. Des études prospectives d'envergure tenant compte de facteurs confusionnels sociodémographiques s'imposent pour éliminer le manque d'uniformité, particulièrement dans le domaine de l'exposition aux métaux lourds, où les éléments de preuve d'un lien avec le TSA demeurent non concluants.

Comme il existe tout un éventail de liens avec le TSA, l'étiologie de ce dernier est probablement multifactorielle. Les études futures devraient continuer à chercher à déterminer les modalités d'interdépendance de ces différents facteurs. Dans l'ensemble, le manque d'uniformité, de temporalité et de spécificité de ces liens entre facteurs environnementaux et TSA demeure l'obstacle le plus important à l'établissement de relations de cause à effet. 


\section{Références}

1. American Psychiatric Association. Diagnostic and Statistical Manual of Mental Disorders: DSM-IV-TR. Washington, DC: American Psychiatric Association; 2000.

2. Boyle CA, Boulet S, Schieve LA, Cohen RA, Blumberg SJ, Yeargin-Allsopp M, et al. Trends in the prevalence of developmental disabilities in US children, 1997-2008. Pediatrics. 2011; 127(6):1034-1042.

3. Centers for Disease Control and Prevention (CDC). Prevalence of autism spectrum disorder among children aged 8 years - Autism and Developmental Disabilities Monitoring Network, 11 sites, United States, 2010. Surveillance Summaries. MMWR. 2014;63(SS02): $1-21$.

4. Base de données épidémiologiques nationale sur l'étude de l'autisme au Canada. Estimated prevalence of autism spectrum disorders among children 2-14 years of age in three Canadian regions (2003-2010) [Internet]. Kingston (Ont.) : NEDSAC; 2012 [consulté le $1^{\mathrm{er}}$ mars 2016]. En ligne à : http://www .nedsac.ca/sites/webpublish.queensu .ca.nedwww/files/files/Table _Prevalence_2003to2010.pdf

5. Rutter M. Incidence of autism spectrum disorders: changes over time and their meaning. Acta Paediatr. 2005; 94(1):2-15.

6. King M, Bearman P. Diagnostic change and the increased prevalence of autism. Int J Epidemiol. 2009;38(5): 1224-1234.

7. Hertz-Picciotto I, Delwiche L. The rise in autism and the role of age at diagnosis. Epidemiology. 2009;20(1):84-90.

8. Hallmayer J, Cleveland S, Torres A, Phillips J, Cohen B, Torigoe $\mathrm{T}$, et al. Genetic heritability and shared environmental factors among twin pairs with autism. Arch Gen Psychiatry. 2011; 68(11):1095-1102.

9. Wigle DT, Arbuckle TE, Walker M, Wade MG, Liu S, Krewski D. Environmental hazards: evidence for effects on child health. J Toxicol Environ Health B Crit Rev. 2007; 10(1-2):3-39.
10. Currenti SA. Understanding and determining the etiology of autism. Cell Mol Neurobiol. 2010;30(2):161-171.

11. Rossignol DA, Genuis SJ, Frye RE. Environmental toxicants and autism spectrum disorders: a systematic review. Transl Psychiatry. 2014;4:e360.

12. Rossignol DA, Frye RE. A review of research trends in physiological abnormalities in autism spectrum disorders: immune dysregulation, inflammation, oxidative stress, mitochondrial dysfunction and environmental toxicant exposures. Mol Psychiatry. 2012;17(4): 389-401.

13. Guinchat V, Thorsen P, Laurent C, Cans C, Bodeau N, Cohen D. Pre-, peri- and neonatal risk factors for autism. Acta Obstet Gynecol Scand. 2012;91(3):287-300.

14. Landrigan PJ. What causes autism? Exploring the environmental contribution. Curr Opin Pediatr. 2010;22(2): 219-225.

15. Curtis LT, Patel K. Nutritional and environmental approaches to preventing and treating autism and attention deficit hyperactivity disorder (ADHD): a review. J Altern Complement Med. 2008;14(1):79-85.

16. Hughes JR. Update on autism: a review of 1300 reports published in 2008. Epilepsy Behav. 2009;16(4): 569-589.

17. Daudt HM, van Mossel C, Scott SJ. Enhancing the scoping study methodology: a large, inter-professional team's experience with Arksey and O'Malley's framework. BMC Med Res Methodol. 2013;13:48.

18. Windham GC, Sumner A, Li SX, et al. Use of birth certificates to examine maternal occupational exposures and autism spectrum disorders in offspring. Autism Res. 2013;6(1):57-63.

19. McCanlies EC, Fekedulegn D, Mnatsakanova A, Burchfiel CM, Sanderson WT, Charles LE, et al. Parental occupational exposures and autism spectrum disorder. J Autism Dev Disord. 2012;42(11):2323-2334.
20. Roberts EM, English PB, Grether JK, Windham GC, Somberg L, Wolff C. Maternal residence near agricultural pesticide applications and autism spectrum disorders among children in the California Central Valley. Environ Health Perspect. 2007;115(10):14821489.

21. Shelton JF, Hertz-Picciotto I, Pessah IN. Tipping the balance of autism risk: potential mechanisms linking pesticides and autism. Environ Health Perspect. 2012;120(7):944-951.

22. Ash MM, Nelson SJ. Wheeler's dental anatomy, physiology and occlusion. 8th ed. St. Louis: Elsevier; 2003.

23. Bernard S, Enayati A, Redwood L, Roger H, Binstock T. Autism: a novel form of mercury poisoning. Med Hypotheses. 2001;56(4):462-471.

24. Woods JS, Martin MD, Naleway CA, Echeverria D. Urinary porphyrin profiles as a biomarker of mercury exposure: studies on dentists with occupational exposure to mercury vapor. J Toxicol Environ Health. 1993;40(2-3):235-246.

25. Nataf R, Skorupka C, Amet L, Lam A, Springbett A, Lathe R. Porphyrinuria in childhood autistic disorder: implications for environmental toxicity. Toxicol Appl Pharmacol. 2006;214(2):99-108.

26. Geier DA, Geier MR. A prospective assessment of porphyrins in autistic disorders: a potential marker for heavy metal exposure. Neurotox Res. 2006; 10(1):57-64.

27. Woods JS, Armel SE, Fulton DI, et al. Urinary porphyrin excretion in neurotypical and autistic children. Environ Health Perspect. 2010;118(10):14501457.

28. Austin DW, Shandley K. An investigation of porphyrinuria in Australian children with autism. J Toxicol Environ Health A. 2008;71(20):13491351.

29. Youn SI, Jin SH, Kim SH, Lim S. Porphyrinuria in Korean children with autism: correlation with oxidative stress. J Toxicol Environ Health A. 2010;73(10):701-710. 
30. Geier DA, Kern JK, Garver CR, et al. Biomarkers of environmental toxicity and susceptibility in autism. J Neurol Sci. 2009;280(1-2):101-108.

31. Kern JK, Geier DA, Adams JB, Geier MR. A biomarker of mercury bodyburden correlated with diagnostic domain specific clinical symptoms of autism spectrum disorder. Biometals. 2010;23(6):1043-1051 .

32. Palmer RF, Blanchard S, Stein Z, Mandell D, Miller C. Environmental mercury release, special education rates, and autism disorder: an ecological study of Texas. Health Place. 2006;12(2):203-209.

33. Palmer RF, Blanchard S, Wood R. Proximity to point sources of environmental mercury release as a predictor of autism prevalence. Health Place. 2009;15(1):18-24.

34. Holmes AS, Blaxill MF, Haley BE. Reduced levels of mercury in first baby haircuts of autistic children. Int $\mathrm{J}$ Toxicol. 2003;22(4):277-285.

35. Geier DA, Kern JK, Geier MR. A prospective study of prenatal mercury exposure from maternal dental amalgams and autism severity. Acta Neurobiol Exp (Wars). 2009;69(2): 189-197.

36. Rahbar MH, Samms-Vaughan $M$, Loveland KA, Ardjomand-Hessabi M, Chen Z, Bressler J, et al. Seafood consumption and blood mercury concentrations in Jamaican children with and without autism spectrum disorders. Neurotox Res. 2013;23(1):22-38.

37. Blaurock-Busch E, Amin OR, Rabah T. Heavy metals and trace elements in hair and urine of a sample of Arab children with autistic spectrum disorder. Maedica (Buchar). 2011;6(4): 247-257.

38. Cobigo V, Murphy MS, Bielska IA, Ouellette-Kuntz H. Applying Hill's Criteria to the study of autism spectrum disorders and exposure to mercury. J Dev Disabil. 2012;18(1):20-33.

39. Ng DK, Chan $\mathrm{CH}$, Soo MT, Lee RS. Low-level chronic mercury exposure in children and adolescents: metaanalysis. Pediatr Int. 2007;49(1):80-87.
40. Agency for Toxic Substances and Disease Registry (US). Toxicological profile for mercury [Internet]. Atlanta (GA): Agency for Toxic Substances and Disease Registry; 1999 [consulté le $1^{\text {er }}$ mars 2014]. En ligne à : http:// www.atsdr.cdc.gov/toxprofiles/tp46.pdf

41. Pichichero ME, Cernichiari E, Lopreiato J, Treanor J. Mercury concentrations and metabolism in infants receiving vaccines containing thiomersal: a descriptive study. Lancet. 2002;360(9347): 1737-1741.

42. Chang S, Crothers C, Lai S, Lamm S. Pediatric neurobehavioral diseases in Nevada counties with respect to perchlorate in drinking water: an ecological inquiry. Birth Defects Res A Clin Mol Teratol. 2003;67(10):886-892 .

43. St-Hilaire S, Ezike VO, Stryhn H, Thomas MA. An ecological study on childhood autism. Int J Health Geogr. 2012;11:44.

44. Becerra TA, Wilhelm M, Olsen J, Cockburn M, Ritz B. Ambient air pollution and autism in Los Angeles County, California. Environ Health Perspect. 2013;121(3):380-386.

45. Kalkbrenner AE, Daniels JL, Chen JC, Poole C, Emch M, Morrissey J. Perinatal exposure to hazardous air pollutants and autism spectrum disorders at age 8. Epidemiology. 2010; 21(5):631-641.

46. Volk HE, Hertz-Picciotto I, Delwiche L, Lurmann F, McConnell R. Residential proximity to freeways and autism in the CHARGE study. Environ Health Perspect. 2011;119(6):873-877.

47. Volk HE, Lurmann F, Penfold B, HertzPicciotto I, McConnell R. Trafficrelated air pollution, particulate matter, and autism. JAMA Psychiatry. 2013; 70(1):71-77.

48. Windham GC, Zhang L, Gunier R, Croen LA, Grether JK. Autism spectrum disorders in relation to distribution of hazardous air pollutants in the San Francisco Bay area. Environ Health Perspect. 2006;114(9):14381444 .
49. Castro-Giner F, Künzli N, Jacquemin B, Forsberg B, de Cid R, Sunyer J, et al. Traffic-related air pollution, oxidative stress genes, and asthma (ECHRS). Environ Health Perspect. 2009;117(12): 1919-1924.

50. Künzli N, Jerrett M, Garcia-Esteban R, et al. Ambient air pollution and the progression of atherosclerosis in adults. PLoS One. 2010;5(2):e9096. doi: 10.1371/journal.pone.0009096.

51. Block ML, Calderón-Garcidueñas L. Air pollution: mechanisms of neuroinflammation and CNS disease. Trends Neurosci. 2009;32(9):506-516.

52. Wakefield AJ, Murch SH, Anthony A, et al. Ileal-lymphoid-nodular hyperplasia, non-specific colitis, and pervasive developmental disorder in children. Lancet. 1998;351(9103):637-641.

53. Smeeth L, Cook C, Fombonne E, et al. MMR vaccination and pervasive developmental disorders: a case-control study. Lancet. 2004;364(9438): 963-969.

54. DeStefano F, Bhasin TK, Thompson WW, Yeargin-Allsopp M, Boyle C. Age at first measles-mumps-rubella vaccination in children with autism and school-matched control subjects: a population-based study in metropolitan Atlanta. Pediatrics. 2004;113(2): 259-266.

55. Mrozek-Budzyn D, Kieltyka A, Majewska R. Lack of association between measles-mumps-rubella vaccination and autism in children: a case-control study. Pediatr Infect Dis J. 2010;29(5):397-400.

56. Takahashi H, Suzumura S, Shirakizawa F, et al. An epidemiological study on Japanese autism concerning routine childhood immunization history. Jpn J Infect Dis. 2003;56(3):114-117.

57. Uno Y, Uchiyama T, Kurosawa M, Aleksic B, Ozaki N. The combined measles, mumps, and rubella vaccines and the total number of vaccines are not associated with development of autism spectrum disorder: the first case-control study in Asia. Vaccine. 2012;30(28):4292-4298. 
58. Hornig M, Briese T, Buie T, et al. Lack of association between measles virus vaccine and autism with enteropathy: a case-control study. PLoS One. 2008; 3(9):e3140.

59. Uchiyama $\mathrm{T}$, Kurosawa $\mathrm{M}$, Inaba $\mathrm{Y}$. MMR-vaccine and regression in autism spectrum disorders: negative results presented from Japan. J Autism Dev Disord. 2007;37(2):210-217.

60. Baird G, Pickles A, Simonoff E, et al. Measles vaccination and antibody response in autism spectrum disorders. Arch Dis Child. 2008;93(10): 832-837.

61. Chen W, Landau S, Sham P, Fombonne E. No evidence for links between autism, MMR and measles virus. Psychol Med. 2004;34(3):543-553.

62. Honda H, Shimizu Y, Rutter M. No effect of MMR withdrawal on the incidence of autism: a total population study. J Child Psychol Psychiatry. 2005;46(6):572-579.

63. Shamberger RJ. Autism rates associated with nutrition and the WIC program. J Am Coll Nutr. 2011;30(5): 348-353.

64. Demicheli V, Jefferson T, Rivetti A, Price D. Vaccines for measles, mumps and rubella in children. Cochrane Database Syst Rev. 2005;(4)(4): CD004407.

65. Wilson K, Mills E, Ross C, McGowan J, Jadad A. Association of autistic spectrum disorder and the measles, mumps, and rubella vaccine: a systematic review of current epidemiological evidence. Arch Pediatr Adolesc Med. 2003;157(7):628-634.

66. Geier DA, Geier MR. A comparative evaluation of the effects of MMR immunization and mercury doses from thimerosal-containing childhood vaccines on the population prevalence of autism. Med Sci Monit. 2004;10(3): PI33-PI39.

67. Singh VK, Jensen RL. Elevated levels of measles antibodies in children with autism. Pediatr Neurol. 2003;28(4): 292-294.
68. Andrews N, Miller E, Grant A, Stowe J, Osborne V, Taylor B. Thimerosal exposure in infants and developmental disorders: a retrospective cohort study in the United Kingdom does not support a causal association. Pediatrics. 2004;114(3):584-591.

69. Price CS, Thompson WW, Goodson B, et al. Prenatal and infant exposure to thimerosal from vaccines and immunoglobulins and risk of autism. Pediatrics. 2010;126(4):656-664.

70. Hviid A, Stellfeld M, Wohlfahrt J, Melbye M. Association between thimerosal-containing vaccine and autism. JAMA. 2003;290(13):1763-1766.

71. Verstraeten T, Davis RL, DeStefano F, et al. Safety of thimerosal-containing vaccines: a two-phased study of computerized health maintenance organization databases. Pediatrics. 2003;112(5): 1039-1048.

72. Madsen KM, Lauritsen MB, Pedersen $\mathrm{CB}$, et al. Thimerosal and the occurrence of autism: negative ecological evidence from Danish populationbased data. Pediatrics. 2003;112(3 Pt 1):604-606.

73. Schechter R, Grether JK. Continuing increases in autism reported to California's developmental services system: mercury in retrograde. Arch Gen Psychiatry. 2008;65(1):19-24.

74. Stehr-Green P, Tull P, Stellfeld M, Mortenson PB, Simpson D. Autism and thimerosal-containing vaccines: lack of consistent evidence for an association. Am J Prev Med. 2003; 25(2):101-106.

75. Geier DA, Geier MR. A two-phased population epidemiological study of the safety of thimerosal-containing vaccines: a follow-up analysis. Med Sci Monit. 2005;11(4):CR160-170.

76. Geier MR, Geier DA. Neurodevelopmental disorders after thimerosal-containing vaccines: a brief communication. Exp Biol Med (Maywood). 2003;228(6): 660-664.

77. Young HA, Geier DA, Geier MR. Thimerosal exposure in infants and neurodevelopmental disorders: an assessment of computerized medical records in the Vaccine Safety Datalink. J Neurol Sci. 2008;271(1-2):110-118.
78. Doja A, Roberts W. Immunizations and autism: a review of the literature. Can J Neurol Sci. 2006;33(4):341-346.

79. Singh VK, Hanson J. Assessment of metallothionein and antibodies to metallothionein in normal and autistic children having exposure to vaccinederived thimerosal. Pediatr Allergy Immunol. 2006;17(4):291-296.

80. Singh VK, Rivas WH. Detection of antinuclear and antilaminin antibodies in autistic children who received thimerosal-containing vaccines. J Biomed Sci. 2004;11(5):607-610.

81. Miles JH, Takahashi TN. Lack of association between Rh status, Rh immune globulin in pregnancy and autism. Am J Med Genet A. 2007;143A(13):13971407.

82. Croen LA, Matevia M, Yoshida CK, Grether JK. Maternal Rh D status, anti-D immune globulin exposure during pregnancy, and risk of autism spectrum disorders. Am J Obstet Gynecol. 2008;199(3):234.e1-e6.

83. Geier DA, Geier MR. A prospective study of thimerosal-containing Rho(D)immune globulin administration as a risk factor for autistic disorders. J Matern Fetal Neonatal Med. 2007; 20(5):385-390.

84. Geier DA, Mumper E, Gladfelter B, Coleman L, Geier MR. Neurodevelopmental disorders, maternal Rh-negativity, and Rho(D) immune globulins: a multi-center assessment. Neuro Endocrinol Lett. 2008;29(2):272-280.

85. Fallon J. Could one of the most widely prescribed antibiotics amoxicillin/ clavulanate "augmentin" be a risk factor for autism? Med Hypotheses. 2005; 64(2):312-315.

86. Good P. Did acetaminophen provoke the autism epidemic? Altern Med Rev. 2009;14(4):364-372.

87. Harrington RA, Lee LC, Crum RM, Zimmerman AW, Hertz-Picciotto I. Serotonin hypothesis of autism: implications for selective serotonin reuptake inhibitor use during pregnancy. Autism Res. 2013;6(3):149-168. 
88. Gur TL, Kim DR, Epperson CN. Central nervous system effects of prenatal selective serotonin reuptake inhibitors: sensing the signal through the noise. Psychopharmacology (Berl). 2013;227(4):567-582.

89. Croen LA, Grether JK, Yoshida CK, Odouli R, Hendrick V. Antidepressant use during pregnancy and childhood autism spectrum disorders. Arch Gen Psychiatry. 2011;68(11):1104-1112.

90. Rai D, Lee BK, Dalman C, Golding J, Lewis GH, Magnusson C. Parental depression, maternal antidepressant use during pregnancy, and risk of autism spectrum disorders: population based case-control study. BMJ. 2013; 346:f2059.

91. Duncan S. Teratogenesis of sodium valproate. Curr Opin Neurol. 2007; 20(2):175-180.

92. Dufour-Rainfray D, Vourc'h P, Tourlet S, Guilloteau D, Chalon S, Andres CR. Fetal exposure to teratogens: evidence of genes involved in autism. Neurosci Biobehav Rev. 2011;35(5):1254-1265.

93. Roullet FI, Lai JK, Foster JA. In utero exposure to valproic acid and autism-a current review of clinical and animal studies. Neurotoxicol Teratol. 2013;36: 47-56.

94. Christensen J, Grønborg TK, Sørensen MJ, et al. Prenatal valproate exposure and risk of autism spectrum disorders and childhood autism. JAMA. 2013; 309(16):1696-1703.

95. Adams JB, Romdalvik J, Ramanujam VM, Legator MS. Mercury, lead, and zinc in baby teeth of children with autism versus controls. J Toxicol Environ Health A. 2007;70(12): 1046-1051.

96. Atladóttir HÓ, Henriksen TB, Schendel DE, Parner ET. Autism after infection, febrile episodes, and antibiotic use during pregnancy: an exploratory study. Pediatrics. 2012;130(6):e1447e1454.

97. Bauer AZ, Kriebel D. Prenatal and perinatal analgesic exposure and autism: an ecological link. Environ Health. 2013;12:41.
98. Schultz ST, Klonoff-Cohen HS, Wingard DL, Akshoomoff NA, Macera CA, Ji M. Acetaminophen (paracetamol) use, measles-mumps-rubella vaccination, and autistic disorder: the results of a parent survey. Autism. 2008;12(3): 293-307.

99. Schultz ST. Can autism be triggered by acetaminophen activation of the endocannabinoid system? Acta Neurobiol Exp (Wars). 2010;70(2):227-231.

100. Gardener H, Spiegelman D, Buka SL. Prenatal risk factors for autism: comprehensive meta-analysis. $\mathrm{Br} \mathrm{J}$ Psychiatry. 2009;195(1):7-14.

101. Bilder D, Pinborough-Zimmerman J, Miller J, McMahon W. Prenatal, perinatal, and neonatal factors associated with autism spectrum disorders. Pediatrics. 2009;123(5):1293-1300.

102. Burstyn I, Sithole F, Zwaigenbaum L. Troubles du spectre autistique, caractéristiques de la mère et complications obstétricales chez les enfants issus d'une grossesse unique en Alberta (Canada). Maladies chroniques au Canada. 2010;30(4):130-140.

103. Mann JR, McDermott S, Bao H, Hardin J, Gregg A. Pre-eclampsia, birth weight, and autism spectrum disorders. J Autism Dev Disord. 2010;40(5):548-554.

104. Haglund NG, Källén KB. Risk factors for autism and Asperger syndrome. Perinatal factors and migration. Autism. 2011;15(2):163-183.

105. Lee BK, Gardner RM, Dal H, et al. Brief report: maternal smoking during pregnancy and autism spectrum disorders. J Autism Dev Disord. 2012; 42(9):2000-2005.

106. Visser JC, Rommelse N, Vink L, et al. Narrowly versus broadly defined autism spectrum disorders: differences in pre- and perinatal risk factors. J Autism Dev Disord. 2013;43(7): 1505-1516.

107. Tran PL, Lehti V, Lampi KM, Helenius $\mathrm{H}$, Suominen A, Gissler $M$, et al. Smoking during pregnancy and risk of autism spectrum disorder in a Finnish national birth cohort. Paediatr Perinat Epidemiol. 2013;27(3):266-274.
108. Kalkbrenner AE, Braun JM, Durkin MS, et al. Maternal smoking during pregnancy and the prevalence of autism spectrum disorders, using data from the Autism and Developmental Disabilities Monitoring Network. Environ Health Perspect. 2012;120(7):1042-1048.

109. Zhang X, Lv CC, Tian J, Miao RJ, Xi W, Hertz-Picciotto I, et al. Prenatal and perinatal risk factors for autism in China. J Autism Dev Disord. 2010;40(11):1311-1321.

110. Eliasen M, Tolstrup JS, Nybo Andersen AM, Grønbaek M, Olsen J, Strandberg-Larsen K. Prenatal alcohol exposure and autistic spectrum disorders--a population-based prospective study of 80,552 children and their mothers. Int J Epidemiol. 2010; 39(4):1074-1081.

111. Mukherjee R, Layton M, Yacoub E, Turk J. Autism and autistic traits in people exposed to heavy prenatal alcohol: data from a clinical series of 21 individuals and nested case control study. Adv Ment Health Intellect Disabil. 2011;5(1):42-49.

112. Kolevzon A, Gross R, Reichenberg A. Prenatal and perinatal risk factors for autism: a review and integration of findings. Arch Pediatr Adolesc Med. 2007;161(4):326-333.

113. Hultman CM, Sandin S, Levine SZ, Lichtenstein P, Reichenberg A. Advancing paternal age and risk of autism: new evidence from a population-based study and a meta-analysis of epidemiological studies. Mol Psychiatry. 2011;16(12):1203-1212.

114. Sandin S, Hultman CM, Kolevzon A, Gross R, MacCabe JH, Reichenberg A. Advancing maternal age is associated with increasing risk for autism: a review and meta-analysis. J Am Acad Child Adolesc Psychiatry. 2012; 51(5):477-486.e1.

115. Frans EM, Sandin S, Reichenberg A, et al. Autism risk across generations: a population-based study of advancing grandpaternal and paternal age. JAMA Psychiatry. 2013;70(5):516-521. 
116. van Balkom ID, Bresnahan M, Vuijk PJ, Hubert J, Susser E, Hoek HW. Paternal age and risk of autism in an ethnically diverse, non-industrialized setting: Aruba. PLoS One. 2012;7(9): e45090.

117. Parner ET, Baron-Cohen S, Lauritsen $\mathrm{MB}$, et al. Parental age and autism spectrum disorders. Ann Epidemiol. 2012;22(3):143-150.

118. Rahbar MH, Samms-Vaughan M, Loveland KA, et al. Maternal and paternal age are jointly associated with childhood autism in Jamaica. J Autism Dev Disord. 2012;42(9): 1928-1938.

119. Pinborough-Zimmerman J, Bilder D, Bakian A, et al. Sociodemographic risk factors associated with autism spectrum disorders and intellectual disability. Autism Res. 2011;4(6): 438-448

120. Kong A, Frigge ML, Masson G, et al. Rate of de novo mutations and the importance of father's age to disease risk. Nature. 2012;488(7412):471-475.

121. Buizer-Voskamp JE, Blauw HM, Boks MP, van Eijk KR, Veldink JH, Hennekam EA, et al. Increased paternal age and the influence on burden of genomic copy number variation in the general population. Hum Genet. 2013;132(4):443-450.

122. Puleo CM, Reichenberg A, Smith CJ, Kryzak LA, Silverman JM. Do autism-related personality traits explain higher paternal age in autism? Mol Psychiatry. 2008;13(3):243-244.

123. Tsuchiya KJ, Matsumoto K, Miyachi $\mathrm{T}$, et al. Paternal age at birth and high-functioning autistic-spectrum disorder in offspring. Br $\mathrm{J}$ Psychiatry. 2008;193(4):316-321.

124. Lyall K, Pauls DL, Spiegelman D, Ascherio A, Santangelo SL. Pregnancy complications and obstetric suboptimality in association with autism spectrum disorders in children of the Nurses' Health Study II. Autism Res. 2012;5(1):21-30.

125. AL-Jammas IK, AL-Dobooni RMY. Prenatal and perinatal risk factors in autistic disorders. Arab J Psychiatr. 2012;23(2):108-114.
126. Durkin MS, Maenner MJ, Newschaffer $\mathrm{CJ}$, et al. Advanced parental age and the risk of autism spectrum disorder. Am J Epidemiol. 2008;168(11): 1268-1276.

127. Croen LA, Najjar DV, Fireman B, Grether JK. Maternal and paternal age and risk of autism spectrum disorders. Arch Pediatr Adolesc Med. 2007;161(4):334-340.

128. Grether JK, Anderson MC, Croen LA, Smith D, Windham GC. Risk of autism and increasing maternal and paternal age in a large North American population. Am J Epidemiol. 2009; 170(9):1118-1126.

129. King MD, Fountain C, Dakhlallah D, Bearman PS. Estimated autism risk and older reproductive age. Am J Public Health. 2009;99(9):1673-1679.

130. Shelton JF, Tancredi DJ, HertzPicciotto I. Independent and dependent contributions of advanced maternal and paternal ages to autism risk. Autism Res. 2010;3(1):30-39.

131. Hvidtjørn D, Grove J, Schendel D, et al. Risk of autism spectrum disorders in children born after assisted conception: a population-based follow-up study. J Epidemiol Community Health. 2011;65(6):497-502.

132. Hvidtjørn D, Schieve L, Schendel D, Jacobsson B, Sværke C, Thorsen P. Cerebral palsy, autism spectrum disorders, and developmental delay in children born after assisted conception: a systematic review and metaanalysis. Arch Pediatr Adolesc Med. 2009;163(1):72-83.

133. Zachor DA, Ben Itzchak E. Assisted reproductive technology and risk for autism spectrum disorder. Res Dev Disabil. 2011;32(6):2950-2956.

134. Shimada T, Kitamoto A, Todokoro A, et al. Parental age and assisted reproductive technology in autism spectrum disorders, attention deficit hyperactivity disorder, and Tourette syndrome in a Japanese population. Res Autism Spectr Disord. 2012;6(1): 500-507.
135. Sandin S, Nygren KG, Iliadou A, Hultman CM, Reichenberg A. Autism and mental retardation among offspring born after in vitro fertilization. JAMA. 2013;310(1):75-84.

136. Lyall K, Pauls DL, Spiegelman D, Santangelo SL, Ascherio A. Fertility therapies, infertility and autism spectrum disorders in the Nurses' Health Study II. Paediatr Perinat Epidemiol. 2012;26(4):361-372.

137. Grether JK, Qian Y, Croughan MS, et al. Is infertility associated with childhood autism? J Autism Dev Disord. 2013 Mar;43(3):663-672.

138. Lehti V, Brown AS, Gissler M, Rihko M, Suominen A, Sourander A. Autism spectrum disorders in IVF children: a national case-control study in Finland. Hum Reprod. 2013;28(3): 812-818.

139. Krakowiak P, Walker CK, Bremer AA, et al. Maternal metabolic conditions and risk for autism and other neurodevelopmental disorders. Pediatrics. 2012;129(5):e1121-e1128.

140. Schrieken M, Visser J, Oosterling I, et al. Head circumference and height abnormalities in autism revisited: the role of pre- and perinatal risk factors. Eur Child Adolesc Psychiatry. 2013; 22(1):35-43.

141. Dodds L, Fell DB, Shea S, Armson BA, Allen AC, Bryson S. The role of prenatal, obstetric and neonatal factors in the development of autism. $\mathrm{J}$ Autism Dev Disord. 2011;41(7): 891-902.

142. Lyall K, Pauls DL, Santangelo SL, Spiegelman D, Ascherio A. Maternal early life factors associated with hormone levels and the risk of having a child with an autism spectrum disorder in the Nurses' Health Study II. J Autism Dev Disord. 2011;41(5): 618-627.

143. Stein D, Weizman A, Ring A, Barak Y. Obstetric complications in individuals diagnosed with autism and in healthy controls. Compr Psychiatry. 2006;47(1):69-75. 
144. Buchmayer S, Johansson S, Johansson A, Hultman CM, Sparen P, Cnattingius $\mathrm{S}$. Can association between preterm birth and autism be explained by maternal or neonatal morbidity? Pediatrics. 2009;124(5):e817-e825.

145. Nath S, Roy R, Mukherjee S. Perinatal complications associated with autism-a case control study in a neurodevelopment and early intervention clinic. J Indian Med Assoc. 2012;110(8):526-529.

146. Hönekopp J. Digit ratio 2D:4D in relation to autism spectrum disorders, empathizing, and systemizing: a quantitative review. Autism Res. 2012;5(4):221-230.

147. de Bruin EI, Verheij F, Wiegman T, Ferdinand RF. Differences in finger length ratio between males with autism, pervasive developmental disorder-not otherwise specified, ADHD, and anxiety disorders. Dev Med Child Neurol. 2006;48(12):962-965.

148. Whitehouse AJ, Mattes E, Maybery MT, Dissanayake C, Sawyer M, Jones $\mathrm{RM}$, et al. Perinatal testosterone exposure and autistic-like traits in the general population: a longitudinal pregnancy-cohort study. J Neurodev Disord. 2012;4(1):25.

149. de Cock M, Maas YG, van de Bor M. Does perinatal exposure to endocrine disruptors induce autism spectrum and attention deficit hyperactivity disorders? Review. Acta Paediatr. 2012;101(8):811-818.

150. Kellman R. The thyroid-autism connection. Exceptional Parent. 2010; 40(4):16.

151. Román GC. Autism: transient in utero hypothyroxinemia related to maternal flavonoid ingestion during pregnancy and to other environmental antithyroid agents. J Neurol Sci. 2007; 262(1-2):15-26.

152. Soldin OP, Lai S, Lamm SH, Mosee S. Lack of a relation between human neonatal thyroxine and pediatric neurobehavioral disorders. Thyroid. 2003;13(2):193-198.
153. Hoshiko S, Grether JK, Windham GC, Smith D, Fessel K. Are thyroid hormone concentrations at birth associated with subsequent autism diagnosis? Autism Res. 2011;4(6):456-463.

154. Korzeniewski SJ, Pinto-Martin JA, Whitaker AH, et al. Association between transient hypothyroxinaemia of prematurity and adult autism spectrum disorder in a low-birthweight cohort: an exploratory study. Paediatr Perinat Epidemiol. 2013;27(2): 182-187.

155. Wallace AE, Anderson GM, Dubrow R. Obstetric and parental psychiatric variables as potential predictors of autism severity. J Autism Dev Disord. 2008;38(8):1542-1554

156. Langridge AT, Glasson EJ, Nassar N, et al. Maternal conditions and perinatal characteristics associated with autism spectrum disorder and intellectual disability. PLoS One. 2013; 8(1):e50963.

157. Gardener H, Spiegelman D, Buka SL. Perinatal and neonatal risk factors for autism: a comprehensive meta-analysis. Pediatrics. 2011;128(2):344-355.

158. Losh M, Esserman D, Anckarsäter H, Sullivan PF, Lichtenstein P. Lower birth weight indicates higher risk of autistic traits in discordant twin pairs. Psychol Med. 2012;42(5): 1091-1102.

159. Lampi KM, Lehtonen L, Tran PL, et al. Risk of autism spectrum disorders in low birth weight and small for gestational age infants. J Pediatr. 2012; 161(5):830-836.

160. Ben Itzchak E, Lahat E, Zachor DA. Advanced parental ages and low birth weight in autism spectrum disorders-rates and effect on functioning. Res Dev Disabil. 2011;32(5): 1776-1781.

161. Leavey A, Zwaigenbaum L, Heavner K, Burstyn I. Gestational age at birth and risk of autism spectrum disorders in Alberta, Canada. J Pediatr. 2013;162(2):361-368.

162. Treyvaud K, Ure A, Doyle LW, et al. Psychiatric outcomes at age seven for very preterm children: rates and predictors. J Child Psychol Psychiatry. 2013;54(7):772-779.
163. Movsas TZ, Paneth N. The effect of gestational age on symptom severity in children with autism spectrum disorder. J Autism Dev Disord. 2012; 42(11):2431-2439.

164. Moore GS, Kneitel AW, Walker CK, Gilbert WM, Xing G. Autism risk in small- and large-for-gestational-age infants. Am J Obstet Gynecol. 2012; 206(4):314.e1-e9.

165. Abel KM, Dalman C, Svensson AC, et al. Deviance in fetal growth and risk of autism spectrum disorder. Am J Psychiatry. 2013;170(4):391-398.

166. Brimacombe $M$, Ming X, Lamendola M. Prenatal and birth complications in autism. Matern Child Health J. 2007;11(1):73-79.

167. Tudor ME, DeVincent CJ, Gadow KD. Prenatal pregnancy complications and psychiatric symptoms: Children with ASD versus clinic controls. Res Autism Spectr Disord. 2012;6(4): 1401-1405.

168. Glasson EJ, Bower C, Petterson B, de Klerk N, Chaney G, Hallmayer JF. Perinatal factors and the development of autism: a population study. Arch Gen Psychiatry. 2004;61(6): 618-627.

169. Gillberg C, Cederlund M. Asperger syndrome: familial and pre- and perinatal factors. J Autism Dev Disord. 2005;35(2):159-166

170. Burstyn I, Wang X, Yasui Y, Sithole F, Zwaigenbaum L. Autism spectrum disorders and fetal hypoxia in a population-based cohort: accounting for missing exposures via EstimationMaximization algorithm. BMC Med Res Methodol. 2011;11:2.

171. Schmidt K, Zimmerman A, Bauman $M$, et al. Brief report: Asperger's syndrome and sibling birth order. J Autism Dev Disord. 2013;43(4): 973-977.

172. Leonard H, Glasson E, Nassar N, et al. Autism and intellectual disability are differentially related to sociodemographic background at birth. PLoS One. 2011;6(3):e17875. 
173. Reichenberg A, Smith C, Schmeidler J, Silverman JM. Birth order effects on autism symptom domains. Psychiatry Res. 2007;150(2):199-204.

174. Martin LA, Horriat NL. The effects of birth order and birth interval on the phenotypic expression of autism spectrum disorder. PLoS One. 2012;7(11): e51049.

175. Cheslack-Postava K, Liu K, Bearman PS. Closely spaced pregnancies are associated with increased odds of autism in California sibling births. Pediatrics. 2011;127(2):246-253.

176. Williams K, Helmer M, Duncan GW, Peat JK, Mellis CM. Perinatal and maternal risk factors for autism spectrum disorders in New South Wales, Australia. Child Care Health Dev. 2008;34(2):249-256

177. Van Naarden Braun K, Schieve L, Daniels J, et al. Relationships between multiple births and autism spectrum disorders, cerebral palsy, and intellectual disabilities: Autism and Developmental Disabilities Monitoring (ADDM) Network-2002 surveillance year. Autism Res. 2008;1(5):266-274.

178. Zerbo O, Iosif AM, Delwiche L, Walker C, Hertz-Picciotto I. Month of conception and risk of autism. Epidemiology. 2011;22(4):469-475.

179. Hebert KJ, Miller LL, Joinson CJ. Association of autistic spectrum disorder with season of birth and conception in a UK cohort. Autism Res. 2010;3(4):185-190.

180. Lee LC, Newschaffer CJ, Lessler JT, Lee BK, Shah R, Zimmerman AW. Variation in season of birth in singleton and multiple births concordant for autism spectrum disorders. Paediatr Perinat Epidemiol. 2008;22(2):172-179.

181. Mazumdar S, Liu KY, Susser E, Bearman P. The disappearing seasonality of autism conceptions in California. PLoS One. 2012;7(7):e41265.

182. Cohly HH, Panja A. Immunological findings in autism. Int Rev Neurobiol. 2005;71:317-341.
183. Grether JK, Croen LA, Anderson MC, Nelson KB, Yolken RH. Neonatally measured immunoglobulins and risk of autism. Autism Res. 2010;3(6): 323-332.

184. Croen LA, Braunschweig D, Haapanen $\mathrm{L}$, et al. Maternal mid-pregnancy autoantibodies to fetal brain protein: the early markers for autism study. Biol Psychiatry. 2008;64(7):583-588.

185. Braunschweig D, Ashwood P, Krakowiak P, et al. Autism: maternally derived antibodies specific for fetal brain proteins. Neurotoxicology. 2008;29(2):226-231.

186. Braunschweig D, Krakowiak P, Duncanson P, et al. Autism-specific maternal autoantibodies recognize critical proteins in developing brain. Transl Psychiatry. 2013;3:e277.

187. Ramaekers VT, Blau N, Sequeira JM, Nassogne MC, Quadros EV. Folate receptor autoimmunity and cerebral folate deficiency in low-functioning autism with neurological deficits. Neuropediatrics. 2007;38(6):276-281.

188. Rothenberg SP, da Costa MP, Sequeira JM, et al. Autoantibodies against folate receptors in women with a pregnancy complicated by a neural-tube defect. N Engl J Med. 2004;350(2): 134-142.

189. Ramaekers VT, Quadros EV, Sequeira JM. Role of folate receptor autoantibodies in infantile autism. Mol Psychiatry. 2013;18(3):270-271.

190. Atladóttir HO, Pedersen MG, Thorsen $P$, et al. Association of family history of autoimmune diseases and autism spectrum disorders. Pediatrics. 2009; 124(2):687-694.

191. Molloy CA, Morrow AL, MeinzenDerr J, et al. Familial autoimmune thyroid disease as a risk factor for regression in children with autism spectrum disorder: a CPEA Study. J Autism Dev Disord. 2006;36(3): 317-324.

192. Croen LA, Grether JK, Yoshida CK, Odouli R, Van de Water J. Maternal autoimmune diseases, asthma and allergies, and childhood autism spectrum disorders: a case-control study. Arch Pediatr Adolesc Med. 2005; 159(2):151-157.
193. Margutti P, Delunardo F, Ortona E. Autoantibodies associated with psychiatric disorders. Curr Neurovasc Res. 2006;3(2):149-157.

194. Vincent A, Dalton P, Clover L, Palace J, Lang B. Antibodies to neuronal targets in neurological and psychiatric diseases. Ann N Y Acad Sci. 2003; 992:48-55.

195. Fox E, Amaral D, Van de Water J. Maternal and fetal antibrain antibodies in development and disease. Dev Neurobiol. 2012;72(10):13271334.

196. Ashwood P, Van de Water J. Is autism an autoimmune disease? Autoimmun Rev. 2004;3(7-8):557-562.

197. Dietert RR, Dietert JM. Potential for early-life immune insult including developmental immunotoxicity in autism and autism spectrum disorders: focus on critical windows of immune vulnerability. J Toxicol Environ Health B Crit Rev. 2008;11(8): 660-680.

198. Hagberg H, Gressens P, Mallard C. Inflammation during fetal and neonatal life: implications for neurologic and neuropsychiatric disease in children and adults. Ann Neurol. 2012; 71(4):444-457.

199. Blaylock RL, Strunecka A. Immuneglutamatergic dysfunction as a central mechanism of the autism spectrum disorders. Curr Med Chem. 2009;16(2):157-170.

200. Blaylock RL. A possible central mechanism in autism spectrum disorders, part 3: the role of excitotoxin food additives and the synergistic effects of other environmental toxins. Altern Ther Health Med. 2009;15(2): 56-60.

201. Abdallah MW, Larsen N, Grove J, Nørgaard-Pedersen B, Thorsen P, Mortensen EL, et al. Amniotic fluid chemokines and autism spectrum disorders: an exploratory study utilizing a Danish Historic Birth Cohort. Brain Behav Immun. 2012;26(1): $170-176$ 
202. Chez MG, Dowling T, Patel PB, Khanna P, Kominsky M. Elevation of tumor necrosis factor-alpha in cerebrospinal fluid of autistic children. Pediatr Neurol. 2007;36(6):361-365.

203. Ashwood P, Krakowiak P, HertzPicciotto I, Hansen R, Pessah IN, Van de Water J. Altered $\mathrm{T}$ cell responses in children with autism. Brain Behav Immun. 2011;25(5):840-849.

204. Abdallah MW, Larsen N, Mortensen EL, et al. Neonatal levels of cytokines and risk of autism spectrum disorders: an exploratory register-based historic birth cohort study utilizing the Danish Newborn Screening Biobank. J Neuroimmunol. 2012; 252(1-2):75-82.

205. Abdallah MW, Larsen N, Grove J, et al. Neonatal chemokine levels and risk of autism spectrum disorders: findings from a Danish historic birth cohort follow-up study. Cytokine. 2013;61(2):370-376.

206. Brown AS. Epidemiologic studies of exposure to prenatal infection and risk of schizophrenia and autism. Dev Neurobiol. 2012;72(10):1272-1276.

207. Zerbo O, Iosif AM, Walker C, Ozonoff $\mathrm{S}$, Hansen RL, Hertz-Picciotto I. Is maternal influenza or fever during pregnancy associated with autism or developmental delays? Results from the CHARGE (CHildhood Autism Risks from Genetics and Environment) study. J Autism Dev Disord. 2013; 43(1):25-33.

208. Maimburg RD, Vaeth M. Perinatal risk factors and infantile autism. Acta Psychiatr Scand. 2006;114(4):257-264.

209. Maimburg RD, Vaeth M, Schendel $\mathrm{DE}$, Bech $\mathrm{BH}$, Olsen J, Thorsen P. Neonatal jaundice: a risk factor for infantile autism? Paediatr Perinat Epidemiol. 2008;22(6):562-568.

210. Maimburg RD, Bech BH, Væth $M$, Møller-Madsen B, Olsen J. Neonatal jaundice, autism, and other disorders of psychological development. Pediatrics. 2010;126(5):872-878.

211. Croen LA, Yoshida CK, Odouli R, Newman TB. Neonatal hyperbilirubinemia and risk of autism spectrum disorders. Pediatrics. 2005;115(2): e135-e138.
212. Zhang Y, Xu Q, Liu J, Li S, Xu X. Risk factors for autistic regression: results of an ambispective cohort study. J Child Neurol. 2012;27(8):975-981.

213. Saemundsen E, Ludvigsson $P$, Rafnsson V. Risk of autism spectrum disorders after infantile spasms: a population-based study nested in a cohort with seizures in the first year of life. Epilepsia. 2008;49(11):18651870.

214. Numis AL, Major P, Montenegro MA, Muzykewicz DA, Pulsifer MB, Thiele EA. Identification of risk factors for autism spectrum disorders in tuberous sclerosis complex. Neurology. 2011;76(11):981-987.

215. Vergani L, Cristina L, Paola R, et al. Metals, metallothioneins and oxidative stress in blood of autistic children. Res Autism Spectr Disord. 2011; 5(1):286-293.

216. Obrenovich ME, Shamberger RJ, Lonsdale D. Altered heavy metals and transketolase found in autistic spectrum disorder. Biol Trace Elem Res. 2011;144(1-3):475-486.

217. El-Ansary A, Al-Daihan S, Al-Dbass A, Al-Ayadhi L. Measurement of selected ions related to oxidative stress and energy metabolism in Saudi autistic children. Clin Biochem. 2010;43(1-2):63-70.

218. Parellada M, Moreno C, Mac-Dowell $\mathrm{K}$, et al. Plasma antioxidant capacity is reduced in Asperger syndrome. $\mathrm{J}$ Psychiatr Res. 2012;46(3):394-401.

219. Blaurock-Busch E, Amin OR, Dessoki HH, Rabah T. Toxic metals and essential elements in hair and severity of symptoms among children with autism. Maedica (Buchar). 2012; $7(1): 38-48$

220. Lakshmi Priya MD, Geetha A. Level of trace elements (copper, zinc, magnesium and selenium) and toxic elements (lead and mercury) in the hair and nail of children with autism. Biol Trace Elem Res. 2011;142(2):148-158.

221. Yasuda H, Yoshida K, Yasuda Y, Tsutsui T. Infantile zinc deficiency: association with autism spectrum disorders. Sci Rep. 2011;1:129.
222. Al-Farsi YM, Waly MI, Al-Sharbati $\mathrm{MM}$, et al. Levels of heavy metals and essential minerals in hair samples of children with autism in Oman: a case-control study. Biol Trace Elem Res. 2013;151(2):181-186.

223. Al-Ayadhi LY. Heavy metals and trace elements in hair samples of autistic children in central Saudi Arabia. Neurosciences (Riyadh). 2005;10(3): 213-218.

224. Chen MH, Su TP, Chen YS, et al. Association between psychiatric disorders and iron deficiency anemia among children and adolescents: a nationwide population-based study. BMC Psychiatry. 2013;13:161.

225. De Palma G, Catalani S, Franco A, Brighenti M, Apostoli P. Lack of correlation between metallic elements analyzed in hair by ICP-MS and autism. J Autism Dev Disord. 2012; 42(3):342-353.

226. Wright B, Pearce H, Allgar V, et al. A comparison of urinary mercury between children with autism spectrum disorders and control children. PLoS One. 2012;7(2):e29547.

227. Meguid NA, Hashish AF, Anwar M, Sidhom G. Reduced serum levels of 25-hydroxy and 1,25-dihydroxy vitamin D in Egyptian children with autism. J Altern Complement Med. 2010;16(6):641-645.

228. Yorbik O, Kurt I, Hasimi A, Oztürk O. Chromium, cadmium, and lead levels in urine of children with autism and typically developing controls. Biol Trace Elem Res. 2010;135(1-3):10-15.

229. Abdullah MM, Ly AR, Goldberg WA, et al. Heavy metal in children's tooth enamel: related to autism and disruptive behaviors? J Autism Dev Disord. 2012;42(6):929-936.

230. Kočovská E, Fernell E, Billstedt E, Minnis H, Gillberg C. Vitamin D and autism: clinical review. Res Dev Disabil. 2012;33(5):1541-1550.

231. DeLuca GC, Kimball SM, Kolasinski J, Ramagopalan SV, Ebers GC. Review: the role of vitamin $\mathrm{D}$ in nervous system health and disease. Neuropathol Appl Neurobiol. 2013; 39(5):458-484. 
232. Grant WB, Soles CM. Epidemiologic evidence supporting the role of maternal vitamin $\mathrm{D}$ deficiency as a risk factor for the development of infantile autism. Dermatoendocrinol. 2009;1(4):223-228.

233. Dealberto MJ. Prevalence of autism according to maternal immigrant status and ethnic origin. Acta Psychiatr Scand. 2011;123(5):339-348.

234. Fernell E, Barnevik-Olsson M, Bågenholm G, Gillberg C, Gustafsson $S$, Sääf M. Serum levels of 25-hydroxyvitamin D in mothers of Swedish and of Somali origin who have children with and without autism. Acta Paediatr. 2010;99(5): 743-747.

235. Whitehouse AJ, Holt BJ, Serralha M, Holt PG, Hart PH, Kusel MM. Maternal vitamin D levels and the autism phenotype among offspring. J Autism Dev Disord. 2013;43(7): 1495-1504.

236. Al-Farsi YM, Al-Sharbati MM, Waly MI, et al. Effect of suboptimal breastfeeding on occurrence of autism: a case-control study. Nutrition. 2012; 28(7-8):e27-e32.

237. Schultz ST, Klonoff-Cohen HS, Wingard DL, et al. Breastfeeding, infant formula supplementation, and autistic disorder: the results of a parent survey. Int Breastfeed J. 2006;1:16.

238. Surén $\mathrm{P}$, Roth $\mathrm{C}$, Bresnahan $\mathrm{M}$, et al. Association between maternal use of folic acid supplements and risk of autism spectrum disorders in children. JAMA. 2013;309(6):570-577.

239. Beard CM, Panser LA, Katusic SK. Is excess folic acid supplementation a risk factor for autism? Med Hypotheses. 2011;77(1):15-17.

240. Maenner MJ, Arneson CL, Durkin MS. Socioeconomic disparity in the prevalence of autism spectrum disorder in Wisconsin. WMJ. 2009;108(5): 253-255.

241. Thomas P, Zahorodny W, Peng B, et al. The association of autism diagnosis with socioeconomic status. Autism. 2012;16(2):201-213.
242. Bhasin TK, Schendel D. Sociodemographic risk factors for autism in a US metropolitan area. J Autism Dev Disord. 2007;37(4):667-677.

243. Emerson E. Deprivation, ethnicity and the prevalence of intellectual and developmental disabilities. J Epidemiol Community Health. 2012;66(3): 218-224

244. Rai D, Lewis G, Lundberg M, et al. Parental socioeconomic status and risk of offspring autism spectrum disorders in a Swedish population-based study. J Am Acad Child Adolesc Psychiatry. 2012;51(5):467-476.e6.

245. Van Meter KC, Christiansen LE, Delwiche LD, Azari R, Carpenter TE, Hertz-Picciotto I. Geographic distribution of autism in California: a retrospective birth cohort analysis. Autism Res. 2010;3(1):19-29.

246. Mandell DS, Wiggins LD, Carpenter LA, et al. Racial/ethnic disparities in the identification of children with autism spectrum disorders. Am J Public Health. 2009;99(3):493-498.

247. Keen DV, Reid FD, Arnone D. Autism, ethnicity and maternal immigration. Br J Psychiatry. 2010;196(4):274-281.

248. Williams PG, Hersh JH, Allard AM, Sears LL. A controlled study of mercury levels in hair samples of children with autism as compared to their typically developing siblings. Res Autism Spectr Disord. 2008;2(1): $170-175$

249. Magnusson C, Rai D, Goodman A, et al. Migration and autism spectrum disorder: population-based study. $\mathrm{Br}$ J Psychiatry. 2012;201:109-115.

250. van der Ven E, Termorshuizen F, Laan W, Breetvelt EJ, van Os J, Selten JP. An incidence study of diagnosed autism-spectrum disorders among immigrants to the Netherlands. Acta Psychiatr Scand. 2013;128(1):54-60.

251. Fountain C, Bearman P. Risk as social context: immigration policy and autism in California. Sociol Forum (Randolph N J). 2011;26(2):215-240.
252. Kinney DK, Munir KM, Crowley DJ, Miller AM. Prenatal stress and risk for autism. Neurosci Biobehav Rev. 2008;32(8):1519-1532.

253. O’Donnell K, O’Connor TG, Glover V. Prenatal stress and neurodevelopment of the child: focus on the HPA axis and role of the placenta. Dev Neurosci. 2009;31(4):285-292.

254. Roberts AL, Lyall K, Rich-Edwards JW, Ascherio A, Weisskopf MG. Association of maternal exposure to childhood abuse with elevated risk for autism in offspring. JAMA Psychiatry. 2013;70(5):508-515.

255. Rai D, Golding J, Magnusson C, Steer C, Lewis G, Dalman C. Prenatal and early life exposure to stressful life events and risk of autism spectrum disorders: population-based studies in Sweden and England. PLoS One. 2012;7(6):e38893.

256. Grether JK, Li SX, Yoshida CK, Croen LA. Antenatal ultrasound and risk of autism spectrum disorders. J Autism Dev Disord. 2010;40(2):238-245.

257. Lathe R. Microwave electromagnetic radiation and autism. E J Appl Psychol. 2009;5(1):11-30.

258. Stoch YK, Williams CJ, Granich J, et al. Are prenatal ultrasound scans associated with the autism phenotype? Follow-up of a randomised controlled trial. J Autism Dev Disord. 2012;42(12):2693-2701.

259. Golding J, Steer C, Pembrey M. Parental and grandparental ages in the autistic spectrum disorders: a birth cohort study. PLoS One. 2010;5(4): e9939.

260. Majewska MD, Urbanowicz E, RokBujko P, Namyslowska I, Mierzejewski P. Age-dependent lower or higher levels of hair mercury in autistic children than in healthy controls. Acta Neurobiol Exp (Wars). 2010;70(2): 196-208

261. Geier DA, Audhya T, Kern JK, Geier MR. Blood mercury levels in autism spectrum disorder: is there a threshold level? Acta Neurobiol Exp (Wars). 2010;70:177-186. 
262. DeSoto MC, Hitlan RT. Blood levels of mercury are related to diagnosis of autism: a reanalysis of an important data set. J Child Neurol. 2007;22(11): 1308-1311.

263. Fido A, Al-Saad S. Toxic trace elements in the hair of children with autism. Autism. 2005;9(3):290-298.

264. Geier DA, Kern JK, King PG, Sykes LK, Geier MR. Hair toxic metal concentrations and autism spectrum disorder severity in young children. Int J Environ Res Public Health. 2012; 9(12):4486-4497.

265. Williams PG, Hersh JH, Allard AM, Sears LL. A controlled study of mercury levels in hair samples of children with autism as compared to their typically developing siblings. Res Autism Spectr Disord. 2008;2(1):170-175.

266. Albizzati A, Morè L, Di Candia D, Saccani M, Lenti C. Normal concentrations of heavy metals in autistic spectrum disorders. Minerva Pediatr. 2012;64(1):27-31.

267. Kern JK, Grannemann BD, Trivedi MH, Adams JB. Sulfhydryl-reactive metals in autism. J Toxicol Environ Health A. 2007;70(8):715-721.

268. El-Ansary AK, Bacha AB, Ayadhi LY. Relationship between chronic lead toxicity and plasma neurotransmitters in autistic patients from Saudi Arabia. Clin Biochem. 2011;44(13):1116-1120.

269. Santé Canada. Rapport sur la biosurveillance humaine des substances chimiques de l'environnement au Canada. Résultats de l'Enquête canadienne sur les mesures de la santé Cycle 1 (2007 à 2009) [Internet]. Ottawa (Ont.) : Ministre de la Santé (Canada); 2010 [consulté le $1^{\mathrm{er}}$ mars 2014]. En ligne à : http://www.hc-sc.gc.ca/ewh -semt/alt_formats/hecs-sesc/pdf /pubs/contaminants/chms-ecms /report-rapport-fra.pdf

270. Santé Canada. Évaluation des risques pour la santé liés au mercure présent dans le poisson et bienfaits pour la santé associés à la consommation de poisson [Internet]. Ottawa (Ont.) : Ministre de la Santé (Canada); 2007 [consulté le $1^{\mathrm{er}}$ mars 2014]. En ligne à : http://publications.gc.ca/collections /collection_2007/hc-sc/H164-54 -2007F.pdf
271. Agency for Toxic Substances and Disease Registry (É.-U.). Toxicological profile for aluminum [Internet]. Atlanta (Géorgie) : Agency for Toxic Substances and Disease Registry; 2008 [consulté le $1^{\mathrm{er}}$ mars 2014]. En ligne à : http://www.atsdr.cdc.gov/toxprofiles /tp22.pdf

272. Agency for Toxic Substances and Disease Registry (É.-U.). Toxicological profile for lead [Internet]. Atlanta (Géorgie) : Agency for Toxic Substances and Disease Registry; 2007 [consulté le $1^{\mathrm{er}}$ mars 2014]. En ligne à : http:// www.atsdr.cdc.gov/toxprofiles/tp13.pdf

273. Agency for Toxic Substances and Disease Registry (É.-U.). Toxicological profile for arsenic [Internet]. Atlanta (Géorgie) : Agency for Toxic Substances and Disease Registry; 2007 [consulté le $1^{\mathrm{er}}$ mars 2014]. En ligne à : http:// www.atsdr.cdc.gov/toxprofiles/tp2.pdf 